\title{
Halbkäfigverbindungen aus Diels-Alder-Addukten des Benzvalens mit Cyclopentadien-Derivaten durch Radikalreaktionen mit Thiophenol und Bromtrichlormethan
}

\author{
Silke Freund ${ }^{\text {), Helmut Henneberger }}{ }^{2)}$ und Manfred Christl* \\ Institut für Organische Chemie der Universität Würzburg, \\ Am Hubland, D-8700 Würzburg \\ Eingegangen am 20. April 1988
}

In Diels-Alder-Reaktionen wurden aus Benzvalen (1) und Tetrachlorcyclopentadienon-dimethylacetal sowie Tetrachlorfuran die Addukte 2 bzw. 8 und 9 erhalten. Behandlung von 2 mit Natrium und tert-Butylalkohol ergab das chlorfreie Acetal 3, dessen Doppelbindung sich mit Diimin unter Bildung von 4 absättigen ließ. Aus dem bekannten Stammkoblenwasserstoff 5 ging analog 6 hervor, und durch Reaktion seines Anions mit Chlorameisensäure-methylester entstand der Ester 7. - Die Verbindungen 2, 3, 5, 7-9 und das bekannte 12 wurden mit Thiophenol so behandelt, da $B$ Radikalkettenreaktionen abliefen. Bei 8 trat Addition an die Zentralbindung des Bicyclobutan-Systems ein. Dagegen unterlag bei 2, 9, 12 und zum Teil bei 3, deren Doppelbindung jeweils der Bicyclobutan-Einheit benachbart ist, das Primärradikal der 5-Hexen-1-yl-Cyclopentylmethyl-Umlagerung, aus der letztlich 15,11,13 bzw. 16 resultierten. Zum Teil bei 3 und vollstãndig bei 5 erfolgte die Addition an die Doppelbindung zu 17 bzw. 18, d.h. ohne Beteiligung der benachbarten Bicyclobutan-Einheit. Durch Aktivierung des Bicyclobutan-Systems mit einer Esterfunktion konnte dessen Passivität aufgehoben werden. So entstanden aus 7 das nichtumgelagerte Produkt 19 und das umgelagerte 20. Ihr Verhältnis hing von der Konzentration an Thiophenol ab. - Analog zur Reaktion 12 + Thiophenol $\rightarrow 13$ brachte Bromtrichlormethan aus 12 das umlagerte Addukt 21 hervor. - Als Nebenprodukt wurde bei der Umsetzung von 12 mit Thiophenol das Chlorwasserstoff-Addukt 14 des ersteren gefunden. Modellreaktionen für die Bildung von 14 sind die Umsetzungen von 12 mit Essigsäure und von 3 sowie 5 mit 4-Nitrobenzoesäure zu den Addukten 22, 23 bzw. 24.

Seit einigen Jahren gewinnen die früher als wenig selektiv geltenden Radikalreaktionen in der Synthese organischer Verbindungen mehr und mehr an Bedeutung ${ }^{3)}$. Als besonders nützlich erweist sich dabei die Cyclisierung des 5-Hexen-1-ylradikals (A) zum Cyclopentylmethylradikal $(\mathbf{B})^{4}$, durch die die Darstellung cyclopentanoider Moleküle wesentlich erleichert wurde ${ }^{3 !}$.
A
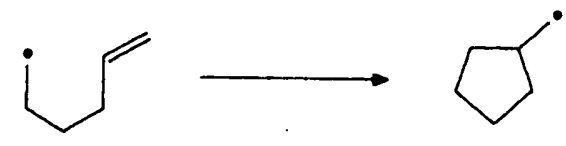

B
Bicyclo[1.1.0]butane addieren Thiole im Rahmen von Radikalkettenreaktionen unter Bildung von Cyclobutylthioethern ${ }^{\text {s) }}$, was zur Synthese zahlreicher Cyclobutan-Derivate genutzt worden ist ${ }^{6}$.
Half-Cage Compounds by Radical Reactions with Thiophenol and Bromotrichloromethane from Diels-Alder Adducts of Benzralene with Cyclopentadiene Derivatives

The adducts 2, 8, and 9 were obtained by Diels-Alder reactions of benzvalene (1) with tetrachlorocyclopentadienone dimethyl acetal and tetrachlorofuran, respectively. On treatment of 2 with sodium and tert-butyl alcohol, the chlorine atoms were substituted by hydrogen atoms to give acetal 3 , the double bond of which could be hydrogenated by diimine with formation of 4 . Analogously, the known parent hydrocarbon 5 afforded 6. The anion of 5 reacted with methyl chloroformate to give the ester 7. - Compounds 2, 3, 5, 7-9, and the known 12 were treated with thiophenol under conditions causing radical chain reactions. Whereas 8 added the reagent across the central bond of the bicyclobutane system, the additions to $2,9,12$ and in part to 3 , the double bond of which is adjacent to the bicyclobutane moiety in each case, were accompanied by a 5-hexen-1-ylcylcopentylmethyl rearrangement of the primary radical resulting in formation of 15,11,13, and 16, respectively. In part with 3 and completely with 5 , the addition took place at the double bond to give 17 and 18, respectively, i.e. without involvement of the adjacent bicylcobutane moiety. Activation of the bicyclobutane system with an ester functionality led to its participation. Thus, compound 7 gave the nonrearranged product 19 and the rearranged one 20 with their ratio being dependent on the concentration of thiophenol. - In analogy to the reaction $12+$ thiophenol $\rightarrow 13$, the rearranged adduct 21 was formed from 12 and bromotrichloromethane. - The hydrogen chloride adduct 14 was found as a byproduct on treatment 12 with thiophenol. Model reactions for the formation of 14 are the additions of acetic acid to 12 and 4-nitrobenzoic acid to 3 and 5 leading to the adducts 22, 23, and 24 , respectively.

Diels-Alder-Reaktionen des Benzvalens mit cyclischen Dienen liefern Produkte, deren Doppelbindung zum Bicyclobutan-System cisorientiert ist ". Mit substituierten Cyclopentadienen entsteht der Verbindungstyp $\mathbf{C}$.

Wenn nun ein Thiylradikal die Zentralbindung der BicyclobutanEinheit von $\mathbf{C}$ von der sterisch günstigeren Seite her angreift, was dem üblichen Verlauf entspricht ${ }^{5.6)}$, sollte das Cyclobutylradikal D entstehen, das sich als Derivat von A entpuppt, in dem Radikalzentrum und Doppelbindung in räumlicher Nähe zueinander festgehalten sind. Die Cyclisierung zum Abkömmling $\mathbf{E}$ von $\mathbf{B}$ ist daher zu erwarten. Zwei Reaktionen, deren Produkte $\mathbf{F}$ aus je einem $\mathbf{R a}$ dikal $\mathbf{E}$ durch H-Abstraktion hervorgehen, haben wir kurz erwähnt ${ }^{7 b}$. Andererseits wurden auch zwei Beispiele dokumentiert, bei denen Pentacyclen $\mathbf{C}$ mit $\mathbf{R}^{\prime}=\mathbf{R}^{3}=\mathrm{H}$ das Thiylradikal an der Doppelbindung zum Radikal $\mathbf{G}$ aufnehmen ${ }^{7 b}$. Die Strukturen der Produkte $\mathbf{H}$ zeigen, daß von $\mathbf{G}$ aus der transannulare Ringschluß 
durch Addition des Radikalzentrums an das Bicyclobutan-System zu $l$ in besagten Fällen nicht stattindet.
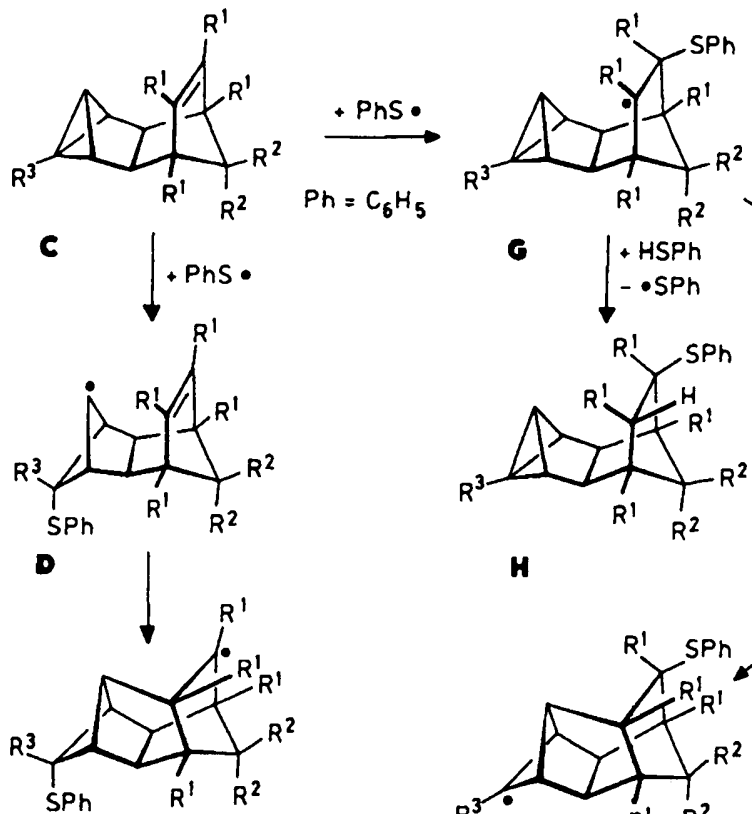

H
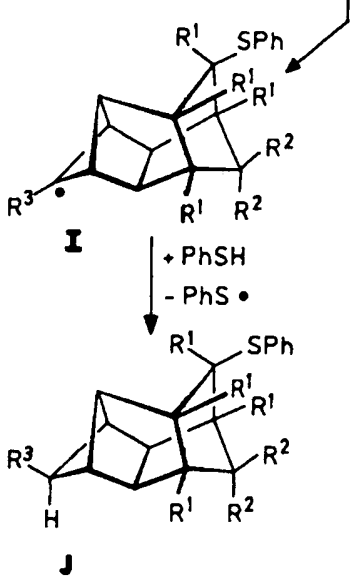

F

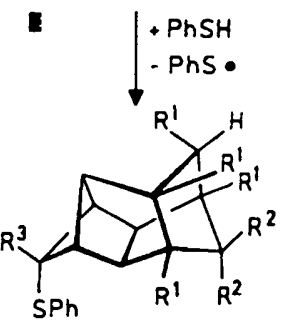

Wir berichten jetzt über Einzelheiten dieser Reaktionen und neue Beispiele, über die Ursache, warum die genannten $\mathbf{G}$ nicht in I übergehen und wie eine solche Umwandlung doch bewirkt werden kann. Das resultierende Produkt $\mathbf{J}$ hat das gleiche Ringgerüst wie F (Decahydro-2,4,6-methenocyclobuta[a]pentalen oder Pentacyclo$\left[6.3 .0 .0^{2.5} \cdot 0^{3.7} \cdot 0^{6.10}\right]$ undecan). Uber dieses Ringsystem ist bisher wenig bekannt. Es wurde lediglich eine Krafteldrechnung des Stammkohlenwasserstoffs zwecks Ermittlung der Spannungsenergie durchgeführt ${ }^{8}$. Die Reaktionsfolge Benzvalen $(\mathbf{1}) \rightarrow \mathbf{C} \rightarrow \mathbf{F}$ bietet einen einfachen Weg zu diesem Ringsystem, und aus den Verbindungen 13 und 15 sollte auch der Stammkohlenwasserstoff durch Routineoperationen zugänglich sein. Weiterhin teilen wir mit, daß auch Bromtrichlormethan analog $\mathrm{zu}$ Thiophenol an eine Verbindung des Typs $\mathbf{C}$ addiert wurde.

\section{A. Darstellung der Edukte}

Die Reaktion von Hexachlorcyclopentadien mit Benzvalen (1) zum Addukt 12 und dessen Reduktion zum Kohlenwasserstoff 5 haben wir bereits beschrieben ${ }^{7 a)}$. In einer Zusammenfassung $^{\text {b) }}$ wurden die Diels-Alder-Reaktion von Tetrachlorcyclopentadienon-dimethylacetal mit Benzvalen (1) zu 2 (61\% Ausbeute) und dessen Reduktion mit Natrium und tert-Butylalkohol zu 3 (79\%) erwähnt. Die Umsetzung von 2 mit Lithium in Ethylendiamin, von der wir neben dem Ersatz der Chlor- durch Wasserstoffatome die reduktive Öfnung der zentralen Bicyclobutan-Bindung ${ }^{9}$ erwarteten, lie- ferte kein einheitliches Produkt. Die Anwendung dieser Bedingungen auf 3 wandelte dieses in das gesättigte Acetal 4 um $(27 \%)$, eine Reaktion, die auch mit Diimin erzielt wurde $(54 \%)$. Zu Vergleichszwecken haben wir den Stammkohlenwasserstoff 6 aus dem Olefin $5^{\text {7a) }}$ dargestellt $(29 \%)$. Das dideuterierte Diels-Alder-Addukt $2 \mathrm{a}$ ging glatt aus [1,6- $\left.\mathrm{D}_{2}\right]$ Benzvalen (1a) und Tetrachlorcyclopentadienon-dimethylacetal hervor.

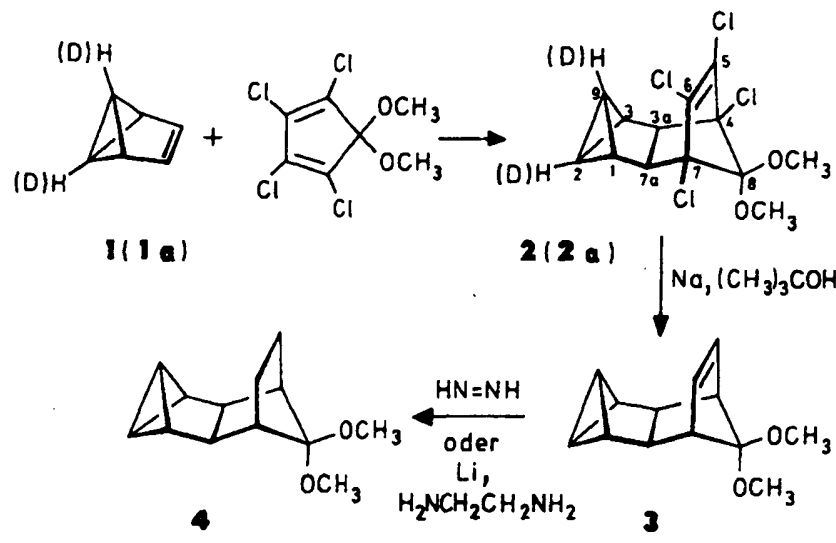

In Anlehnung an das Verfahren zur Bereitung von Tricyclo[4.1.0.0.0.7. ${ }^{2,7}$ heptan-1-carbonsäure-methylester ${ }^{10)}$ führten wir 5 durch Metallierung mit $n$-Butyllithium in Gegenwart von Tetramethylethylendiamin und anschließende Zugabe von Chlorameisensäure-methylester in den Ester 7 über $(24 \%)$.

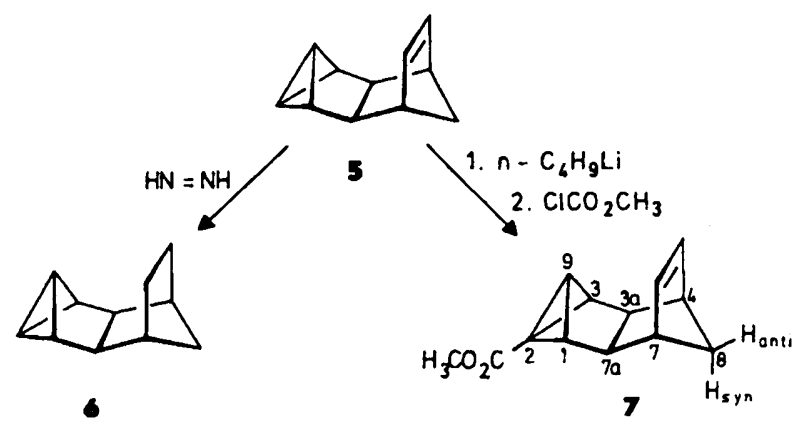

Eine neue Diels-Alder-Reaktion des Benzvalens (1) gelang mit Tetrachlorfuran, das als bewährte Dien-Komponente in $[4+2]$-Cycloadditionen bekannt ist ${ }^{11,12)}$. Es lieferte ein 1.0:3.6-Gemisch der isomeren Addukte 8 und $9(81 \%)$, die durch fraktionierende Kristallisation getrennt wurden. 9 und 10 bildeten sich unter kinetischer Kontrolle, d.h. die Reaktionen sind nicht reversibel, so da $\mathrm{B}$ die Aldersche endoRegel ${ }^{13)}$ weniger streng erfüllt ist als bei der Anlagerung von Hexachlorcyclopentadien an $1^{\text {7a) }}$.

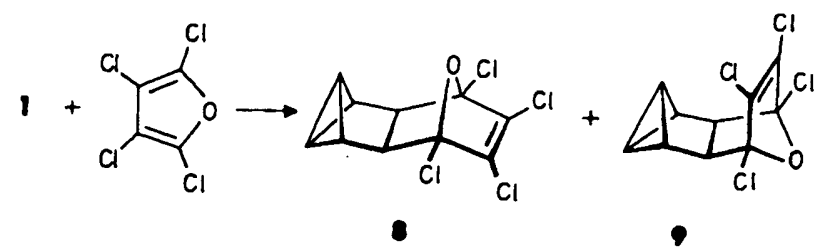

Chem. Ber. 121, 1665-1674 (1988) 
Während die Natur obiger Verbindungen als Tricyclo[3.1.0.0 ${ }^{26}$ ] hexan-Derivate eindeutig aus den NMR-Spektren folgt (Tab. 1 und 2), schließen wir auf die relative Orientierung von Bicyclobutan-System und Doppelbindung aufgrund der Umsetzungen mit Thiophenol, die bei 2, 3, 7 und 9, nicht aber bei 8 transannulare Reaktionen auslösten (siehe Abschnitt B). Die Position der Esterfunktion in 7 leiten wir aus dem Protonen-gekoppelten ${ }^{13} \mathrm{C}$-NMR-Spektrum ab, in dem die Feinstruktur des Signals des unsubstituierten Bicyclobutan-Brückenkopfs aus einem Triplett mit $8 \mathrm{~Hz}$ besteht. Aufgrund eines früher entwickelten Kriteriums ${ }^{60.79}$ sollte es sich dabei um ${ }^{3} J_{\mathrm{C}-9,3 \mathrm{a}-\mathrm{H}}$ handeln (antiperiplanare Bindungsanordnung). Eine Feinstruktur des Signals des substituierten Bicyclobutan-Brückenkopfs von 7 ist dagegen nicht aufgelöst.

\section{B. Umsetzungen mit Thiophenol}

Die Reaktionen der Tetrachlorofuran-Addukte 8 und 9 mit Thiophenol in Benzol bei $80^{\circ} \mathrm{C}$ mit Azoisobutyronitril (AIBN) als Radikalstarter, bei 9 auch bei $20^{\circ} \mathrm{C}$ unter Belichten, nahmen einen sehr unterschiedlichen Verlauf. Während bei 8 lediglich die Anlagerung an die zentrale Bicyclobutan-Bindung zu 10 (38\% Ausbeute) eintrat, wurden bei 9 sowohl das Bicyclobutan-System als auch die Dichlorvinyleneinheit verändert, und dem Produkt schreiben wir die Struktur 11 (24, 21\%, analog Typ F) zu. Damit findet nach der Addition des Thiylradikals an 9 zu einem Cyclobutylradikal analog zu Typ $\mathbf{D}$ der $\mathbf{D} \rightarrow \mathbf{E}$ entsprechende Umlagerungsschritt statt, bevor von Thiophenol ein H-Atom übernommen wird.

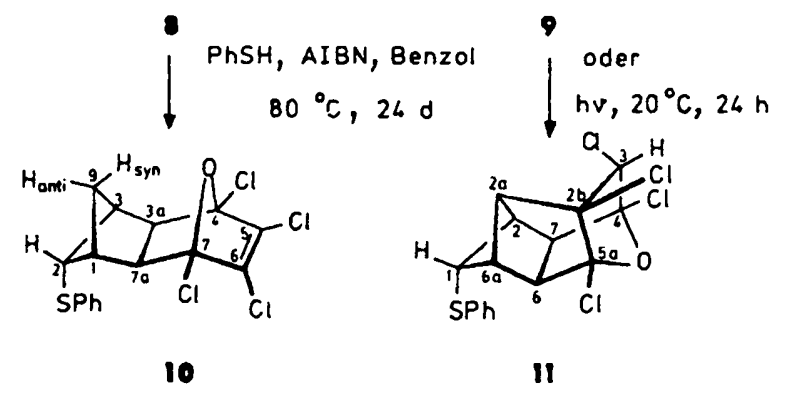

Für die Struktur 10 sprechen die ${ }^{13} \mathrm{C}$-NMR-Signale olefinischer $\mathrm{C}$-Atome $(\delta=138.1)$ und die durch ${ }^{1} \mathrm{H}$ - und ${ }^{13} \mathrm{C}$ NMR-Spektren belegte $C_{s}$-Symmetrie. Die Stellung des Phenylthiosubstituenten an $\mathrm{C}-2$ und nicht an $\mathrm{C}-9$, woraus sich der Angriff des Radikals von der sterisch günstigeren Seite ableitet, folgt aus ${ }^{4} J_{3 a, 9 a n t i}=1.3 \mathrm{~Hz}$ (W-Anordnung der Bindungen zwischen diesen Kernen) und aus dem Fehlen von ${ }^{4} J_{2,3 \mathrm{a}}$. Die NMR-Spektren von 11 (Tab. 3 und 4) beweisen dessen Asymmetrie und die Abwesenheit olefinischer CAtome. Die Kopplungskonstanten im 'H-NMR-Spektrum stehen mit den Interplanarwinkeln am Ringgerüst von 11 im Einklang. So unterscheiden sich $J_{2,7}=1.3$ und $J_{6,6 \mathrm{a}}=$ $1.7 \mathrm{~Hz}$ wegen ungleicher Winkel, die am Modell zu ca. 70 bzw. $55^{\circ}$ bestimmt wurden, meßbar (vgl. Tab. 3). Aus ${ }^{4} J_{3.7}=$ $1.0 \mathrm{~Hz}$ (W-Anordnung der Bindungen zwischen diesen Kernen) schließen wir auf die Konfiguration an C-3.

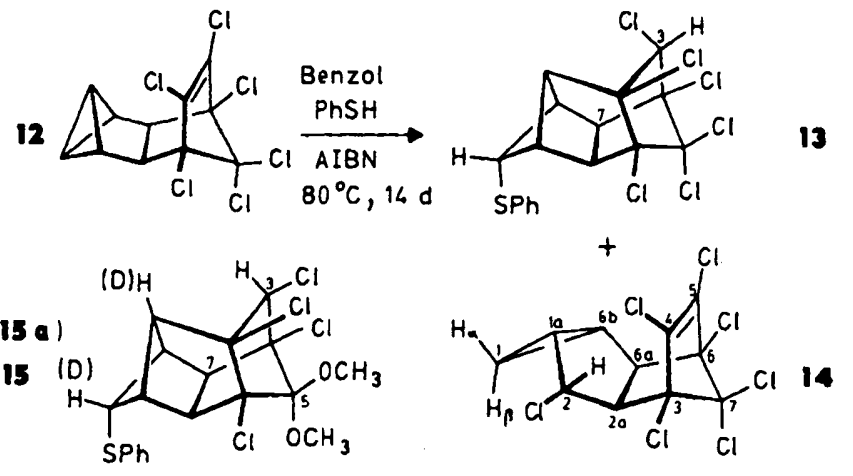

Auch die Umsetzung der Addukte 12 $^{\text {7al }}$ und 2 von Hexachlorcyclopentadien bzw. Tetrachlorcyclopentadienondimethylacetal an Benzvalen mit Thiophenol unter Bedingungen, bei denen Thiylradikale generiert werden, führte zu umgelagerten Produkten, die strukturell 11 ähnlich sind, nämlich 13 und 15 ( 23 bzw. 66\%, Typ F). Neben 13 fanden wir die Heptachlorverbindung 14, die wohl durch Addition von Chlorwasserstoff an 12 entstanden war. Vermutlich verursachte die lange Reaktionszeit den Zerfall eines Teils von 12 unter Bildung von Chlorwasserstoff. Zum Vergleich wurden 3, 5 und 12 mit Säuren behandelt und zu 14 analoge Produkte erhalten (vgl. Abschnitt D).

Mit der Absicht, die noch nicht umgelagerte Radikalzwischenstufe des Typs $\mathbf{D}$ abzufangen, wurde eine Lösung von 2 in reinem Thiophenol belichtet. $\mathrm{Daß}$ sich wieder nur reines 15 ergab, zeigt die hohe Geschwindigkeit des Cyclisierungsschritts $\mathbf{D} \rightarrow \mathbf{E}$ an. Das dideuterierte Substrat 2a lieferte das spezifisch dideuterierte Produkt 15a, dessen 'H-NMR-Spektren im Vergleich zu dem von 15 die Zuordnung der Signale erleichterte.

Die Konfiguration an C-3 von 13 wird wie bei 11 durch die Kopplungskonstante ${ }^{4} J_{3.7}=1.0 \mathrm{~Hz}$ angezeigt. Als Indiz für die invertierte Konfiguration werten wir das Fehlen dieser Wechselwirkung bei 15. Wahrscheinlich schirmt die benachbarte Methoxygruppe an C-5 des Radikals vom Typ E die $\beta$-Seite von $C-3$ sterisch so weit $a b$, daß das Wasserstoffatom im Gegensatz zur Situation bei der Bildung von 11 und 13 nur von der $\alpha$-Seite her übernommen werden kann.

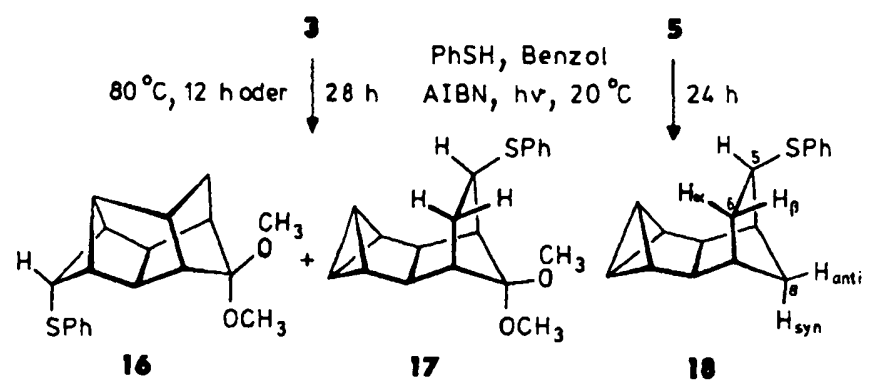

Anders als 2 brachte das chlorfreie Acetal 3 mit Thiophenol sowohl beim Erhitzen als auch beim Belichten ein Gemisch (35\%) hervor, das aus den Addukten 16 und 17 im Verhältnis nahe $1: 1$ bestand. Die zu 17 analoge Verbindung 18 war das einzige Produkt (58\%) der Belichtung einer ben- 
zolischen Lösung von Thiophenol und dem formalen Cyclopentadien-Addukt 5 an Benzvalen. Die denkbare Anlagerung eines weiteren Thiophenol-Moleküls jeweils an das Bicyclobutan-System von 17 und 18 haben wir nicht beobachtet, sollte aber unter energischeren Bedingungen erzielbar sein (vgl. die Bildung des 2:1-Addukts von Thiophenol an Benzvalen ${ }^{(4)}$ ).

Während 16 und 18 vollständig charakterisiert sind, stützt sich die Struktur von 17 nur auf die 'H-NMR-Signale der beiden Methoxygruppen und von 5-H. Die Absorption des letzteren weist wie bei 18 aufgrund der Kopplungskonstanten auf seine endo-Position am Norbornan-System.

Somit läuft bei 5 nur die Anlagerung von Thiophenol an die Vinyleneinheit $a b$, beginnend mit der Addition eines Phenylthiylradikals von der exo-Seite her wie bei Norbornen-Derivaten üblich. Bereits im Falle von 3 konkurriert dieser Prozeß erfolgreich mit der Reaktion am Bicyclobutan-System. Was ist nun die Ursache dafür, daß die Substrate 2, 8, 9 und 12 vom Phenylthiylradikal ausschließlich an der Bicyclobutan-Einheit angegriffen werden, während bei 5 die Doppelbindung reaktiver ist und 3 eine Mittelstellung einnimmt? Wie die Ergebnisse der Umsetzungen verschiedener Bicyclobutan-Derivate ${ }^{s c l}$, auch der von Benzvalen ${ }^{(4)}$ und Homobenzvalen ${ }^{6 d .14)}$ mit Thiophenol zeigen, unterliegen diese Radikalkettenreaktionen der Orbitalkontrolle. Das scheint auch bei obigen Substraten zuzutreffen, d.h. das elektrophile Phenylthiylradikal attackiert jeweils am Ort des HOMOs. Wie die ersten Ionisationspotentiale der Modellsubstanzen für 5, Norbornen (8.97 $\left.\mathrm{eV}^{(5)}\right)$ und Tricyclo[3.1.0.0 $0^{2.6}$ hexan $\left(9.2 \mathrm{eV}^{(6)}\right)$, demonstrieren, dürfte in 5 das HOMO in der Vinyleneinheit lokalisiert sein. Durch den induktiven Effekt der Methoxygruppen in $\mathbf{3}$ ist wohl der Energieunterschied zwischen dem $\pi$-Orbital der Vinyleneinheit und dem obersten besetzten Orbital des Bicyclobutansystems ( $a_{1}$, in der Zentralbindung lokalisiert) verkleinert. Weiterhin desaktiviert die nachbarständige Methoxygruppe die Doppelbindung aus sterischen Gründen. Die Summe der Wirkungen führt zu einer ähnlichen Reaktivität von Vinylen- und Bicyclobutan-Einheit. Durch den induktiven Effekt senken die Chloratome in 2, 8,9 und 12 die Energie des $\pi$-Orbitals soweit $a b$, daß das HOMO eindeutig zum Bicyclobutan-Teil gehört. Wie die langen Reaktionszeiten dieser Verbindungen im Vergleich mit 3 andeuten, stabilisieren die Chloratome auch das relativ weit entfernte HOMO noch merkbar.

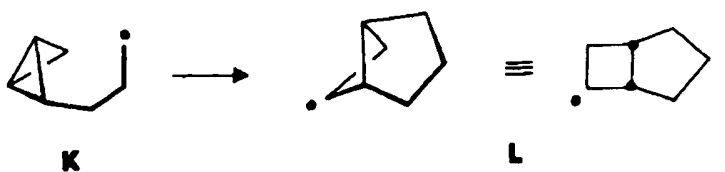

Es stellt sich die weitere Frage, warum bei der Addition des Phenylthiylradikals an die Vinyleneinheit die Cyclisierung des Typs $\mathbf{G} \rightarrow \mathbf{I}$ unterbleibt. Es resultieren ja die Verbindungen 17 und 18, die zum Typ $\mathbf{H}$ gehören. Folgeprodukte $\boldsymbol{J}$ von $Z$ wischenstufen des Typs $I$ haben wir nicht gefunden. Im Gegensatz zur Cyclisierung $\mathbf{A} \rightarrow \mathbf{B}$ der Art $\mathbf{D} \rightarrow \mathbf{E}$ beim Einsatz von 2, 3, 9 und 12 läuft die 3-(endo-2-
Bicyclobutyl)-1-propyl - Bicyclo[3.2.0]hept-6-yl-Radikalumlagerung $(K \rightarrow L)$ der Art $\mathbf{G} \rightarrow I$ im Fall von 3 und 5 nicht ab. Und dies, obwohl die Reaktionsenthalpie für den letzteren Prozeß um ca. $20 \mathrm{kcal} / \mathrm{mol}$ günstiger ist als für den ersteren ${ }^{17}$. Somit ist nach der Ursache für die relativ hohe Aktivierungsenergie der exothermen Umlagerung $\mathbf{G} \rightarrow$ I zu fragen.
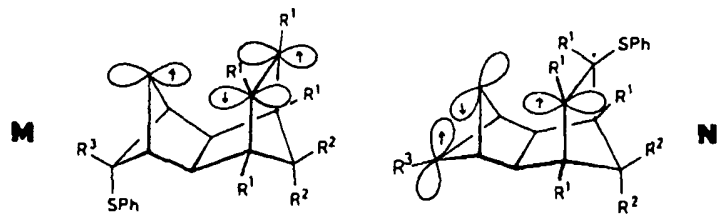

Zwei Möglichkeiten liegen auf der Hand. Zum einen könnte der Abstand zwischen den Orbitalen, die miteinander in Wechselwirkung treten müssen, in $\mathbf{G}$ (siehe Formel $\mathbf{N}$ ) größer sein als in $\mathbf{D}$ (siehe Formel $\mathbf{M}$ ). Im letzteren weist das Orbital mit dem Einzelelektron recht genau in Richtung des $\pi$-Elektronen-Systems der Vinyleneinheit. Dagegen steht in $\mathbf{N}$ das Orbital mit dem Einzelelektron weniger günstig bezüglich des besetzten Orbitals der zentralen BicyclobutanBindung. Das liegt an dieser Bindung, weil sie die Bicyclobutan-Brückenkopf-C-Atome auf Distanz zum NorbornanGerüst hält. Zum anderen könnte der Grund auch elektronischer Natur sein. Da sowohl in $D(M)$ als auch in $\mathbf{G}(\mathbf{N})$ ein C-Radikal vorliegt, das nucleophilen Charakter hat, dürfte die Wechselwirkung mit dem LUMO der Vinylenbzw. Bicyclobutan-Einheit die Reaktionsgeschwindigkeit bestimmen. Trifft diese Hypothese zu, dann müßte das $\sigma^{*}$ Orbital der zentralen Bicyclobutan-Bindung energiereicher sein als das $\pi^{*}$-Orbital der CC-Doppelbindung.

Letztere Vorstellung haben wir experimentell geprüft und gefunden, daß sie die Wirklichkeit korrekt beschreibt, allerdings ohne den Einfluß der unterschiedlichen geometrischen Verhältnisse in $\mathbf{D}(\mathbf{M})$ und $\mathbf{G}(\mathbf{N})$ auszuschließen. So isolierten wir nach Erhitzen des Esters 7 in reinem Thiophenol in Gegenwart von AIBN mit 56\% Ausbeute ein 1.0:0.4Gemisch aus dem nicht umlagerten und dem umlagerten Addukt 19 bzw. 20. Als 7 und Thiophenol unter großer Verdünnung umgesetzt wurden, erhielten wir mit geringer Ausbeute nur noch 20, wobei der maximale Anteil von 19 im Rohprodukt durch das Verhältnis 19:20 $\leqq 1: 18$ ange-

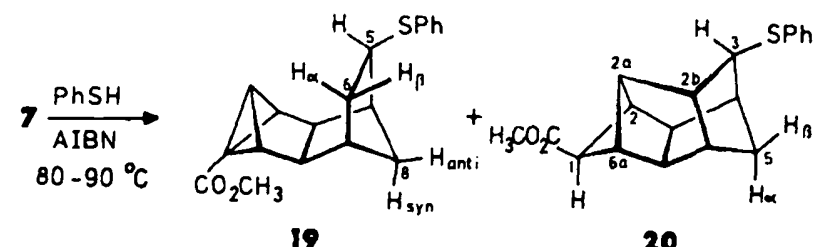

\begin{tabular}{cccc}
\hline mmol 7 & mmol PhSH & ml Benzol & $19: 20$ \\
\hline 0.74 & 48.9 & - & $1.0: 0.4$ \\
0.74 & 7.4 & 6 & $1.0: 0.6$ \\
0.74 & 0.82 & 1.8 & $1.0: 1.7$ \\
0.74 & 0.82 & 500 & $\leqq 1.0: 18$ \\
\hline
\end{tabular}


geben werden kann. Die Zusammenstellung unter den Formeln zeigt, daß zwei Versuche mit weniger extremen Konzentrationen weniger extreme 19:20-Verhältnisse lieferten.

Diese Ergebnisse charakterisieren die Konkurrenzsituation, der das Radikal des Typs $\mathbf{G}$ ausgesetzt ist. Thiophenol in hoher Konzentration fängt es weitgehend zum nicht umgelagerten Produkt 19 des Typs $\mathbf{H}$ ab. Im Falle hoch verdünnten Thiophenols hat es Zeit zur vollständigen Cyclisierung zum Radikal des Typs I, so daB nur noch dessen Abfangprodukt 20 (Typ J) gefunden wird. Der Vergleich der Umsetzungen von 5 und 7 mit Thiophenol beweist die Erhöhung der Reaktivität des Bicyclobutan-Systems (Absenkung des LUMO) durch eine Estergruppe beim intramolekularen Angriff eines Cyclopentylradikals.

Im 'H-NMR-Spektrum von 20 ist die Multiplizität der Signale von $2-\mathrm{H}$ und $6 \mathrm{a}-\mathrm{H}$ niedriger als bei den übrigen Verbindungen der Tab. 3. Wahrscheinlich geht die fehlende Kopplung auf die veränderte Konfiguration an C-1 zurück. Es ist schwer zu beurteilen, von welcher Seite das Radikal des Typs I ein H-Atom von Thiophenol bevorzugt übernimmt. Bei verwandten Radikalen, die aus Tricyclo[4.1.0.0.0.7. heptan-Derivaten ${ }^{\left.S_{2}\right)}$ und Homobenzvalen ${ }^{(d)}$ erzeugt wurden, verläuft dieser Prozeß wenig spezifisch. Somit ist die Konfiguration der Formel 20 durchaus wahrscheinlich. In Analogie zu anderen Derivaten des Bicyclo[2.1.1] hexans ${ }^{\text {(8) }}$ sollten in 20 die Kopplungskonstanten $J_{1,2}$ und $J_{1,6 a}$ nicht aufgelöst sein. DaB das 1-H-Signal dennoch die Triplett-Multiplizität aufweist, könnte durch $2 \mathrm{a}-\mathrm{H}$ und $2 \mathrm{~b}-\mathrm{H}$ verursacht sein, weil die Bindungswege zu diesen Protonen teilweise bzw. vollständig einem Zick-Zack-Weg folgen.

\section{Umsetzung von 12 mit Bromtrichlormethan}

Die homolytische Addition von Bromtrichlormethan an die Doppelbindung von Olefinen ist zur Knüpfung von CCBindungen nutzbar ${ }^{3}$. Wegen der elektronegativen Substituenten ist das Trichlormethylradikal elektrophil ${ }^{3)}$, weshalb wir es als Reaktionspartner für das Bicyclobutan-System in Betracht zogen. Die Belichtung von Bromtrichlormethan in Gegenwart von AIBN und Tricyclo[4.1.0.0.7. unter anderem zur trans-Addition an die zentrale Bicyclobutan-Bindung geführt ${ }^{19}$.

12

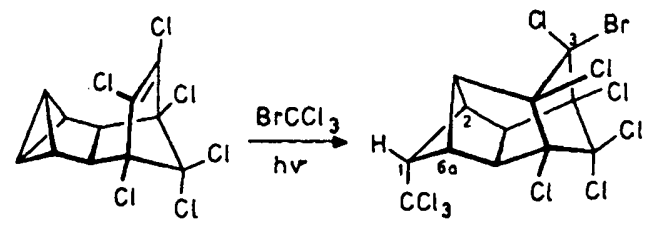

Tatsächlich ergab die Belichtung von Bromtrichlormethan und 12 in Hexan ein 1:1-Addukt (53\%), dem wir Struktur 21 zuschreiben. Während die Konfiguration von $\mathrm{C}-1$ aus der Größe der Kopplungskonstanten $J_{1,2}=J_{1,6 \mathrm{a}}=3.0 \mathrm{~Hz}$ folgt, ist die von C-3 nicht gesichert aber wahrscheinlich. Es steht nicht einmal fest, ob nicht die Substituenten $\mathrm{CCl}_{3}$ und $\mathrm{Br}$ vertauscht sind. Gewöhnlich ist jedoch in Radikalreaktionen von Bromtrichlormethan das Trichlormethylradikal kettentragend ${ }^{3)}$, weshalb wir seine Addition an das Bicy-
clobutan-System (Ort des HOMO) als wahrscheinlich erachten. Das zum Typ D analoge Radikal mäßte dann umlagern und das zum Typ $E$ analoge von Bromtrichlormethan ein Bromatom übernehmen.

\section{Umsetzung von 3, 5 und 12 mit Säuren}

Anla $\beta$ für diese Untersuchungen war die Bildung von 14, dem Chlorwasserstoff-Addukt von 12, das neben dem Thiophenol-Addukt 13 beim zweiwöchigen Rückflußkochen von 12 und Thiophenol in Benzol entstand (Abschnitt B). Vermutlich war dabei ein Teil von 12 unter Freisetzung von Chlorwasserstoff zerfallen. Die Struktur von 14 ist charakteristisch für die Anlagerung einer Säure an ein endo,endoüberbrücktes Bicyclobutan ${ }^{20}$. Insbesondere verläuft die Reaktion von Chlorwasserstoff oder Essigsäure mit Benzvalensulfid vollständig analog ${ }^{21}$. Dabei tritt ein Proton von der sterisch günstigeren Seite an das Bicyclobutan-System unter Retention und Offnung einer der beiden peripheren Bindungen. Das entstehende Kation vom Cyclopropylcarbinyltyp nimmt dann das Nucleophil auf, das das Proton übertragen hat. Da dies wahrscheinlich vom Kontakt-Ionenpaar aus geschieht, resultiert am Cylopropylcarbinyl-CAtom Retention der Konfiguration.

Um zu prüfen, ob dieser Ablauf, wie durch die Bildung von 14 angedeutet, für Verbindungen des Typs $C$ allgemein gilt, haben wir 12 mit Essigsäure behandelt und dabei das Acetat $22(50 \%)$ erhalten. Allerdings gelang die Abtrennung einer Beimengung nicht. Kristalline und daher leichter zu reinigende Präparate erwarteten wir beim Einsatz von 4Nitrobenzoesäure. Dies bestätigte sich auch, da aus 3 und 5 die festen Addukte $23(73 \%)$ bzw. $24(76 \%)$ in reiner Form hervorgingen.

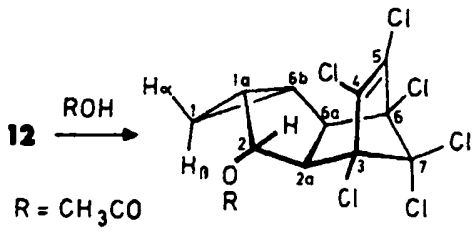

22

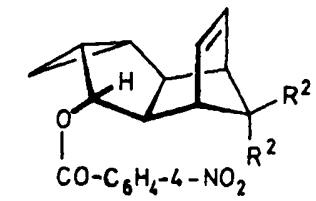

$23\left(R^{2}=\propto H_{3}\right), 24\left(R^{2}=H\right)$
Die Konfiguration der Ringsysteme von 14 und 22-24, d.h. die trans-Anordnung von Dreiring und Norbornen-Einheit am Fünfring, wird ebenso wie die an $\mathrm{C}-2$ durch die ${ }^{1} \mathrm{H}$ NMR-Spektren (Tab. 5) belegt. So spricht die Größe von $J_{1 \mathrm{a}, 2}=5.4-5.6 \mathrm{~Hz}, J_{1 \mathrm{a}, 6 \mathrm{~b}}=4.8-6.0 \mathrm{~Hz}$ sowie $J_{2 \mathrm{a}, 6 \mathrm{~b}}=$ $7.3-7.7 \mathrm{~Hz}$ für die cis-vicinale Stellung der betreffenden Protonen am Fünfring und die von $J_{2,2 \mathrm{a}}=2.4-3.0 \mathrm{~Hz}$ sowie $J_{62,6 b}<1 \mathrm{~Hz}$ für die trans-vicinale Positionen der zugehörigen Protonen. $J_{2 a, 3}=J_{6,6 a}=4.0-4.5 \mathrm{~Hz}$ in 23 und 24 sind für die zweifach endo-substituierte Norbornen-Einheit typisch. Aufgrund der dem Modell entnehmbaren Interplanarwinkel stimmen all diese Werte gut mit der Erwartung überein.

Die Bildung der Verbindungen 14 und 22-24 einerseits und die des Typs $\mathbf{F}, \mathbf{H}$ und $\mathbf{J}$ andererseits charakterisiert die Abläufe über ionische bzw. radikalische $\mathrm{Zwischenstufen}$ 
bei der HX-Addition an Bicyclobutane mit benachbarter, jedoch nicht konjugierter CC-Doppelbindung (Typ C).

Wir danken dem Fonds der Chemischen Industrie für die Förderung, insbesondere für ein Chemiefonds-Stipendium für S. F. Herr Dr. D. Wendisch, Bayer AG, Leverkusen, übernahm dankenswerterweise die Messung des 'H-NMR-Spektrums von 21 und die Entkopplungsexperimente. Unser Dank gilt ferner der Union Rheinische Braunkohlen Kraftstoff $A G$. Wesseling, für Spenden von Dimethylether.

\section{Experimenteller Teil \\ Geräte: Lit. ${ }^{7 c)}$}

\section{4,7-Methano-1,2,3-metheno-1 $H$-inden-Derivate}

( $1 \alpha, 2 \alpha, 3 \alpha, 3 a \beta, 4 \alpha, 7 \alpha, 7 a \beta)-4,5,6,7-$ Tetrachlor-2,3,3a,4,7,7a-hexahydro-8,8-dimethoxy-4,7-methano-1,2,3-metheno-1H-inden (2): Bei $20{ }^{\circ} \mathrm{C}$ löste man $2.33 \mathrm{~g}(8.83 \mathrm{mmol})$ 1,2,3,4-Tetrachlor-5,5-dimethoxycyclopentadien $^{221}$ in einer Lösung von Benzvalen ${ }^{23)}(8.90 \mathrm{mmol})$ in Ether. Nach $3 \mathrm{~d}$ war Benzvalen 'H-NMR-spektroskopisch nicht mehr nachweisbar. Man engte $i$. Vak. ein und destillierte aus dem Rückstand überschüssiges Dien bei $60-70^{\circ} \mathrm{C}(\mathrm{Bad}) / 0.01$ Torr ab. Es blieben $1.85 \mathrm{~g}(61 \%) 2$ als gelber Kristallbrei zurück, aus dem durch Sublimation bei $80-90^{\circ} \mathrm{C} / 0.01$ Torr farblose Kristalle, nach Umlösen aus Ethanol Schmp. $96-98^{\circ} \mathrm{C}$, erhalten wurden. - IR $(\mathrm{KBr}): 1605 \mathrm{~cm}^{-1}(\mathrm{C}=\mathrm{C}) .-\mathrm{MS}(70 \mathrm{eV}): \mathrm{m} / \mathrm{z}(\%)=344,342,340$ $\left(0.6,1.2,0.9, \mathrm{M}^{+}\right), 309,307,305\left(30,94,100, \mathrm{M}^{+}-\mathrm{Cl}\right) .{ }^{1} \mathrm{H}$ NMR: Tab. 1. $-{ }^{13} \mathrm{C}-\mathrm{NMR}$ : Tab. 2.

$$
\begin{array}{llllll}
\mathrm{C}_{13} \mathrm{H}_{12} \mathrm{Cl}_{4} \mathrm{O}_{2} \text { (342.1) } & \text { Ber. C } 45.65 \text { H } 3.54 \mathrm{Cl} 41.46 \\
& \text { Gef. C } 45.51 \text { H } 3.74 \mathrm{Cl} 41.58
\end{array}
$$

[2,9-D ]-Derivat 2a: Darstellung analog zu 2 durch Einsatz von [1,6-D $D_{2}$-Benzvalen ${ }^{24}$. Im 'H-NMR-Spektrum von 2a fehlt im Vergleich $\mathrm{zu}$ dem von 2 das $\mathrm{m}$ bei $\delta=1.88-2.28$.

$(1 \alpha, 2 \alpha, 3 \alpha, 3 a \beta, 4 \beta, 7 \beta, 7 a \beta)-2,3,3 a, 4,7,7 a-H e x a h y d r o-8,8$-dimethoxy-4,7-methano-1,2,3-metheno-1H-inden (3): Unter Stickstoff und Rühren tropfte man zu $7.85 \mathrm{~g}$ (341 mmol) kleingeschnittenem Natrium in $60 \mathrm{ml}$ unter Rückfluß siedendem absol. Tetrahydrofuran innerhalb von $2 \mathrm{~h} 3.50 \mathrm{~g}$ (10.2 mmol) 2 und $11.4 \mathrm{~g}(154 \mathrm{mmol})$ tertButylalkohol in $20 \mathrm{ml}$ absol. Tetrahydrofuran. Dabei färbte sich die Lösung violett. Nach $15 \mathrm{stdg}$. Siedem brachte man überschüssiges Natrium mit $15 \mathrm{ml}$ Methanol in Lösung, gab anschließend so viel Wasser zu, daß sich der Niederschlag gerade löste, sättigte mit $\mathrm{NaCl}$, trennte die Phasen, extrahierte die wäBrige Phase mehrmals mit Tetrahydrofuran, trocknete die vereinigten organischen Phasen über $\mathrm{MgSO}_{4}$ und engte i.Vak. ein. Aus dem gelben Rückstand destillierten bei $60-70^{\circ} \mathrm{C}(\mathrm{Bad}) / 0.7$ Torr $1.65 \mathrm{~g}(79 \%)$ farbloses flüssiges 3. - MS $(70 \mathrm{eV}): m / z(\%)=204\left(15, \mathrm{M}^{+}\right), 157(22), 129(100)$, $128(41), 115(32), 111(30), 95$ (20), 91 (25), 78 (21), 77 (21), 52 (22), 51 (20). - 'H-NMR: Tab. 1. - ${ }^{13}$ C-NMR: Tab. 2.

$$
\begin{array}{lll}
\mathrm{C}_{13} \mathrm{H}_{16} \mathrm{O}_{2} \text { (204.3) } & \text { Ber. C } 76.44 \text { H } 7.90 \\
& \text { Gef. C } 75.96 \text { H } 8.10
\end{array}
$$

( $(\alpha, 2 \alpha, 3 \alpha, 3 a \beta, 4 \beta, 7 \beta, 7 a \beta)-2,3,3 a, 4,5,6,7,7 a-O c t a h y d r o-8,8$-dimethoxy-4,7-methano-1,2,3-metheno-1H-inden (4) aus 3. - a) Mit Lithium in Ethylendiamin: Unter Stickstoff und Rühren löste man $110 \mathrm{mg}(15.9 \mathrm{mmol})$ kleingeschnittenes Lithium in $3.60 \mathrm{~g}(60 \mathrm{mmol})$ Ethylendiamin (blaue Lösung) und tropfte langsam $800 \mathrm{mg}(3.92$ mmol) 3 in $1 \mathrm{ml}$ Ethylendiamin zu. Nach einer Induktionsperiode von einigen Minuten erwärmte sich das Gemisch und schäumte. Am Ende der Zutropfzeit (40 $\mathrm{min}$ ) war das Gemisch grün und veränderte sich durch $5 \mathrm{~min}$. Rückflußkochen nach farblos. Man setzte vorsichtig so viel Wasser $\mathrm{zu}, \mathrm{daB}$ sich der Niederschlag auflöste, extrahierte mehrmals mit Ether, trocknete die vereinigten organischen Phasen mit $\mathrm{MgSO}_{4}$ und engte i. Vak. ein. Aus dem Rückstand destillierten bei $40-50^{\circ} \mathrm{C}$ (Bad)/0.8 Torr $220 \mathrm{mg}(27 \%) 4$ als gelbe, jedoch laut ' $H$-NMR-Spektrum kaum verunreinigte Flüssigkeit.

b) Mit Diimin: $350 \mathrm{mg}(1.71 \mathrm{mmol}) 3$ wurden mit $7.40 \mathrm{~g}$ (34.3 mmol) $p$-Nitrobenzolsulfonsäurehydrazid in $70 \mathrm{ml} 1.5 \mathrm{M}$ Ethanolamin in Methanol ${ }^{25)} 2 \mathrm{~h}$ unter Rückfluß erhitzt. Nach Abkühlen gab man $100 \mathrm{ml}$ Wasser zu und arbeitete wie bei a) auf: $190 \mathrm{mg}$ $(54 \%) 4$ als farblose Flüssigkeit. - MS $(70 \mathrm{eV}): \mathrm{m} / \mathrm{z}(\%)=206(26$, $\mathrm{M}^{+}$), 191 (37), 131 (35), 117 (51), 115 (43), 101 (100), 97 (30), 91 (68), 83 (36), 77 (39), 55 (65), 39 (41). - 'H-NMR: Tab. 1.

$$
\mathrm{C}_{13} \mathrm{H}_{18} \mathrm{O}_{2}(206.3) \text { Ber. C } 75.69 \text { H } 8.80
$$
Gef. C 75.17 H 8.99

( $1 x, 2 x, 3 \alpha, 3 a \beta, 4 \beta, 7 \beta, 7 a \beta)-2,3,3 a, 4,5,6,7,7 a-O c t a h y d r o-4,7-m e-$ thano-1,2,3-metheno- $1 \mathrm{H}$-inden (6): $1.10 \mathrm{~g}(7.42 \mathrm{mmol}) 5^{72)}, 19.8 \mathrm{~g}$ (396 mmol) Hydrazinhydrat und $19.9 \mathrm{~g} \mathrm{(326} \mathrm{mmol)} \mathrm{Ethanolamin}$ in $80 \mathrm{ml}$ Ethylenglycolmonomethylether wurden bei $20^{\prime \prime} \mathrm{C}$ unter kräftigem Rühren innerhalb von $2 \mathrm{~h}$ mit $94.2 \mathrm{~g}(286 \mathrm{mmol})$ $\mathrm{K}_{3} \mathrm{Fe}(\mathrm{CN})_{6}$ in $200 \mathrm{ml}$ Wasser versetzt ${ }^{25}$. Man extrahierte die geibe Mischung fünfmal mit Ether und die vereinigten Etherphasen dreimal mit Wasser, trocknete die Etherphase mit $\mathrm{Na}_{2} \mathrm{SO}_{4}$ und engte i. Vak. ein. Aus dem Rückstand destillierten bei $80-85^{\circ} \mathrm{C}(\mathrm{Bad}) / 15$ Torr $310 \mathrm{mg}(29 \%) 6$ als farblose Flüssigkeit. - MS (70 eV): $\mathrm{m} / \mathbf{z}$ $(\%)=146\left(21, \mathrm{M}^{+}\right), 117(58), 105(73), 91(48), 80(55), 79(56), 78$ (100), 68 (71), 67 (97), 39 (45). - 'H-NMR: Tab. 1. - ${ }^{13}$ C-NMR: Tab. 2.

$$
\begin{array}{lll}
\mathrm{C}_{11} \mathrm{H}_{14} \text { (146.2) } & \text { Ber. C } 90.35 \text { H } 9.65 \\
& \text { Gef. C } 90.59 \text { H } 9.79
\end{array}
$$

(1 $\alpha, 2 \beta, 3 \alpha, 3 a \beta, 4 \beta, 7 \beta, 7 a \beta)-1,3,3 a, 4,7,7 a-$ Hexahydro-4,7-methano1,2,3-metheno-2H-inden-2-carbonsäure-methylester (7): Unter Stickstoff gab man $1.50 \mathrm{ml}(9.90 \mathrm{mmol})$ absol. Tetramethylethylendiamin zu $9.9 \mathrm{mmol}$ eisgekühltem $n$-Butyllithium $(6.6 \mathrm{ml} 1.5 \mathrm{M}$ in Hexan). Nach Entfernen des Kühlbads tropfte man unter kräftigem Rühren $1.43 \mathrm{~g}(9.92 \mathrm{mmol})$ frisch von $\mathrm{LiAlH}_{4}$ abdestilliertes $5^{\text {ia) }}$ in $2 \mathrm{ml}$ absol. $n$-Hexan innerhalb von $10 \mathrm{~min} z$, wobei sich die Mischung gelbbraun färbte. Man rührte $24 \mathrm{~h}$ und tropfte dann die entstandene Suspension nach Aufschlämmung mit $5 \mathrm{ml}$ absol. $n$-Hexan langsam in eine auf $0^{\circ} \mathrm{C}$ gekühlte Lösung von $10.0 \mathrm{~g}(106 \mathrm{mmol})$ frisch destilliertem Chlorameisensäure-methylester in $10 \mathrm{ml}$ absol. $n$-Hexan. Nach $15 \mathrm{~min}$. Rühren lie $B$ man auf $20^{\circ} \mathrm{C}$ kommen, verdünnte mit $10 \mathrm{ml} n$-Hexan, filtrierte einen schmierigen Niederschlag ab und engte das Filtrat i.Vak. ein. Aus dem Rückstand destillierte bei $60-80^{\circ} \mathrm{C}(\mathrm{Bad}) / 15$ Torr überschüssiger Chlorameisensäureester $\mathrm{ab}$, und bei $94-100^{\circ} \mathrm{C}(\mathrm{Bad}) / 0.1$ Torr gingen $490 \mathrm{mg}(24 \%) 7$ als farbloses Öl über. - IR (Film): $1720 \mathrm{~cm}^{-1}(\mathrm{C}=\mathrm{O})$. - MS $(70 \mathrm{eV})$ : $m / z(\%)=202\left(5, \mathrm{M}^{+}\right), 143(22), 136(19), 128(23), 115(18), 105$ (47), $79(73), 77(54), 66(100), 51(20), 39(20)$. - 'H-NMR: Tab. 1. ${ }^{13}$ C-NMR: Tab. 2.

$$
\begin{array}{lll}
\mathrm{C}_{13} \mathrm{H}_{14} \mathrm{O}_{2} \text { (202.3) } & \text { Ber. C } 77.20 \text { H } 6.98 \\
& \text { Gef. C } 77.23 \text { H } 6.90
\end{array}
$$

$(1 \alpha, 2 \alpha, 3 \alpha, 3 a \beta, 4 \beta, 7 \beta, 7 a \beta)-(8)$ und $(1 \alpha, 2 \alpha, 3 \alpha, 3 a \beta, 4 \alpha, 7 \alpha, 7 a \beta)-4,5,6,7-$ Tetrachlor-2,3,3a,4,7,7a-hexahydro-4,7-epoxy-1,2,3-metheno- $1 \mathrm{H}$ inden (9): Tetrachlorfuran wurde aus Perchlorbutadien über Hexachlor-2,5-dihydrofuran ${ }^{26)}$ dargestellt. Bei der Eliminierung von Chlor aus letzterem mit Zink verwendeten wir in Abänderung der Vorschrift ${ }^{27)}$ Natrium- $\beta$-naphthalinsulfonat als Katalysator mit der Konsequenz, daB ein 7:3-Gemisch aus Produkt und Edukt anfiel. - ${ }^{13} \mathrm{C}$-NMR $\left(\mathrm{C}_{6} \mathrm{D}_{6}\right)$ : Hexachlor-2,5-dihydrofuran: $\delta=110.6$, 133.4; Tetrachlorfuran: $\delta=113.8,132.4$.

$2.06 \mathrm{~g}$ (10.0 mmol) Tetrachlorfuran (als Gemisch mit Hexachlordihydrofuran), eine Spatelspitze $\mathrm{K}_{2} \mathrm{CO}_{3}$ und $1.95 \mathrm{~g}$ (25.0 mmol) Benzvalen ${ }^{23)}$ in $65 \mathrm{ml}$ Ether wurden $3 \mathrm{~d}$ bei $20^{\circ} \mathrm{C}$ gerührt (NMR- 
Kontrolle). Nach Filtration engte man i. Vak. ein. Aus dem gelben öligen Rückstand destillierten bei $70-90^{\circ} \mathrm{C}$ (Bad)/0.001 Torr $2.30 \mathrm{~g}$ $(81 \%)$ eines gelblichen 1.0:3.6-Gemisch aus 8 und 9, das zu einem Kristallbrei erstarrte. Durch fraktionierende Kristallisation aus Ether erhielt man $530 \mathrm{mg}(19 \%) 8$ als farblose Kristalle mit Schmp. $140-141^{\circ} \mathrm{C}$ und $1.54 \mathrm{~g}(54 \%) 9$ als schmierige farblose Kristalle mit Schmp. $43-48^{\circ} \mathrm{C}$. - IR (KBr) von 8: $3120,3055,2960(\mathrm{C}-\mathrm{H})$, $1610 \mathrm{~cm}^{-1}(\mathrm{C}=\mathrm{C})$. - MS $(70 \mathrm{eV})$ von $8: \mathrm{m} / \mathrm{z}(\%)=286,284,282$ $\left(0.7,1.5,1.1, \mathrm{M}^{+}\right), 208(32), 206(65), 204(51), 185(38), 183(55), 149$ (32), 78 (100). - 'H-NMR: Tab. 1. - ${ }^{13} \mathrm{C}-\mathrm{NMR}$ : Tab. 2.

$$
\begin{array}{rrrrr}
\mathrm{C}_{10} \mathrm{H}_{6} \mathrm{Cl} & \mathrm{O} \text { (284.0) } & \text { Ber. } \mathrm{C} 42.29 & \mathrm{H} 2.13 \\
\text { 8: } & \text { Gef. } \mathrm{C} 42.34 & \text { H } 1.90 \\
\text { 9: } & \text { Gef. } \mathrm{C} 42.63 & \text { H } 2.19
\end{array}
$$

Tab. 1. 'H-NMR-Chemische Verschiebungen ( $\delta$-Werte) und Kopplungskonstanten $[\mathrm{Hz}]$ von 4,7-Methano-1,2,3-methenoinden-Derivaten in $\mathrm{CDCl}_{3}$. Die generell angegebenen Multiplizitäten sind nur bei Abwesenheit von Effekten höherer Ordnung zu beobachten, was bei $400 \mathrm{MHz}$ meist, bei $90 \mathrm{MHz}(2-4)$ meist nicht zutrifft. Die

\begin{tabular}{|c|c|c|c|c|c|c|}
\hline $\mathrm{Nr}$. & $\begin{array}{c}2-\mathrm{H} \\
\mathrm{dt}\end{array}$ & $\begin{array}{l}\text { 9-H } \\
d d t^{a)}\end{array}$ & $\begin{array}{c}1,3-H \\
\text { br.t }\end{array}$ & $\begin{array}{c}\text { 3a,7a-H } \\
\text { br.s }\end{array}$ & $\begin{array}{l}\text { 4,7-H } \\
\text { m(br.s) }\end{array}$ & $\underset{\mathrm{m}}{5,6-\mathrm{H}}$ \\
\hline $\begin{array}{l}2^{\text {b) }} \\
3^{\text {c) }} \\
4^{\text {(b) }} \\
6^{\text {h) }} \\
7^{\text {hi) }} \\
8 \\
9\end{array}$ & $\begin{array}{c}1.88 \\
1.55^{\mathrm{d}} \\
1.33 \\
1.39 \\
\overline{2} \\
2.26 \\
2.35\end{array}$ & $\begin{array}{l}-2.28 \\
1.88^{\mathrm{d})} \\
-2.15 \\
1.53 \\
2.59^{\mathrm{j}} \\
2.53 \\
2.23\end{array}$ & $\begin{array}{l}2.03 \\
1.83 \\
1.98^{\mathrm{d}} \\
1.83 \\
2.61^{\mathrm{k})} \\
2.17 \\
2.05\end{array}$ & $\begin{array}{l}2.78 \\
2.43 \\
2.01^{\mathrm{d}} \\
2.02^{\mathrm{d})} \\
2.48 \\
2.72 \\
3.17\end{array}$ & $\begin{array}{l}2 . \overline{73^{\mathrm{e}}} \\
2.24 \\
2.10^{\mathrm{d}} \\
2.63^{\prime \prime} \\
- \\
-\end{array}$ & $\begin{array}{l}5 . \overline{86^{n}} \\
1.33-2.15 \\
1.21,1.79 \\
5.83^{1} \\
- \\
-\end{array}$ \\
\hline
\end{tabular}
Kopplungskonstanten variieren nur wenig: $J_{29}=8.1-9.2, J_{12}=$ $1.5-1.8, J_{1.9}=1.5-2.0, J_{3 \mathrm{a}, 9}=1.1-1.5$ (Ausnahme 6: 1.9)

a) Bei ähnlicher Größe von $J_{1,9}$ und $J_{3 \mathrm{a}, 9}$ dquint. - b) 3.53 und 3.57 (jeweils s; $2 \mathrm{CH}_{3}$ ). - c) 3.03 und 3.13 (jeweils s; $2 \mathrm{CH}_{3}$ ). a Zuordnung austauschbar. - e Pseudo-quint mit Linienabstand von etwa $2.1 \mathrm{~Hz}$, durch Entkopplung des Signals von 5,6- $\mathrm{H}$ charakterisiert. - 0 Pseudo-t mit Linienabstand $2.1 \mathrm{~Hz}$ - -8.23 (s) $2 \mathrm{CH}_{3}$ ). - h) 1.79 und 1.99 (jeweils $\mathrm{m}$, überlagert; 8- $\mathrm{H}_{2}$ ). - i) 1.33 $\left(\mathrm{dtt}, J_{8,8}=8.0, J_{4.8 s y n}=1.5, J_{5,8 s y n}=0.7 ; 8-H_{s y n}\right), 1.48\left(\mathrm{dt}, J_{4.8 a n t i}=\right.$ $\left.1.8 ; 8-\mathrm{H}_{\text {anti }}\right)$, 3.56 (s; $\mathrm{CH}_{3}$ ). - ${ }^{3}$ tt. - k) Br.d. - " Pseudo-sept $(4,7-\mathrm{H})$ und br. pseudo-t $(5,6-\mathrm{H})$ mit Linienabstand $1.8 \mathrm{~Hz}$.

\section{Reaktionen mit Thiophenol}

( $1 \alpha, 2 \beta, 3 \alpha, 3 a \beta, 4 \beta, 7 \beta, 7 a \beta)-4,5,6,7-$ Tetrachlor-2,3,3a,4,7,7a-hexahydro-2-phenylthio-4,7-epoxy-1,3-methano- $1 \mathrm{H}$-inden (10): $500 \mathrm{mg}(1.76$ mmol) 8, eine Spatelspitze Azoisobutyronitril (AIBN) und $210 \mathrm{mg}$ (1.91 mmol) Thiophenol wurden in $10 \mathrm{ml}$ Benzol $24 \mathrm{~d}$ unter Rückfluß erhitzt. Danach extrahierte man das Gemisch mit $2 \mathrm{~N} \mathrm{NaOH}$, dann mit Wasser, trocknete mit $\mathrm{Na}_{2} \mathrm{SO}_{4}$ und verdampite das Lösungsmittel i.Vak. Aus dem gelben Rückstand destillierten bei $120-140^{\circ} \mathrm{C}(\mathrm{Bad}) / 0.001$ Torr $261 \mathrm{mg}(38 \%) 10$ als farbloses Ol, das beim Anreiben mit Petrolether Kristalle mit Schmp. $116-118^{\circ} \mathrm{C}$ lieferte. - IR $(\mathrm{KBr}): 1605(\mathrm{CCl}=\mathrm{CCl}), 1585\left(\mathrm{C}_{6} \mathrm{H}_{5}\right) \mathrm{cm}^{-1}$. - MS $(70 \mathrm{eV}): m / z(\%)=398,396,394,392\left(0.1,0.9,1.8,1.4, \mathrm{M}^{+}\right), 249$ (32), 247 (33), 188 (33), 110 (38), 79 (100), 77 (31). - 'H-NMR $\left(\mathrm{CDCl}_{3}\right): \delta=1.44\left(\mathrm{dtt}, J_{9,9}=8.5, J_{1,9 a n t i}=2.5 \mathrm{~Hz}, J_{3 a, 9 a n t i}=\right.$ $\left.1.3 \mathrm{~Hz} ; 9-\mathrm{H}_{\text {anti }}\right), 2.67$ (d; 9- $\left.\mathrm{H}_{s j \mathrm{n}}\right), 2.84(\mathrm{~m} ; 1,3-\mathrm{H}), 2.96$ (br. s; 3a,7aH), $3.66\left(t, J_{1.2}=2.5 \mathrm{~Hz} ; 2-\mathrm{H}\right), \mathrm{C}_{6} \mathrm{H}_{5}: 7.21(p-\mathrm{H}), 7.26-7.30(0-, m-$ H). $-{ }^{13} \mathrm{C}-\mathrm{NMR}\left(\mathrm{CDCl}_{3}\right): \delta=29.5$, (t; C-9), 44.9 (d; C-1,3), 54.3 (d; C-3a,7a), 60.1 (d; C-2), 101.1 (s; C-4,7), 138.1 (s; C-5,6), $\mathrm{C}_{6} \mathrm{H}_{5}$ : 126.7 (p-C), 129.1 und 129.9 (o-, $m-C), 135.4$ (ipso-C).

$$
\begin{array}{rrrr}
\mathrm{C}_{16} \mathrm{H}_{12} \mathrm{Cl}_{4} \mathrm{OS}(394.2) & \text { Ber. C } 48.76 \text { H } 3.07 \\
10: & \text { Gef. C } 48.77 \text { H } 2.96 \\
\text { 11: } & \text { Gef. C } 49.07 \text { H } 3.03
\end{array}
$$

Tab.2. ${ }^{13} \mathrm{C}-\mathrm{NMR}$-Chemische Verschiebungen ( $\delta$-Werte) von 4,7Methano-1,2,3-methenoinden-Derivaten in $\mathrm{CDCl}_{3}$ und bei 6 und 7 auch ${ }^{3} \mathrm{C}, \mathrm{H}$-Kopplungskonstanten $\left(\mathrm{Hz},{ }^{1} J_{\mathrm{CH}}\right.$ in der zweiten Zeile). Wo das Protonen-gekoppelte Spektrum nicht zur Verfügung stand, half ein off-resonance-entkoppeltes bei der Zuordnung

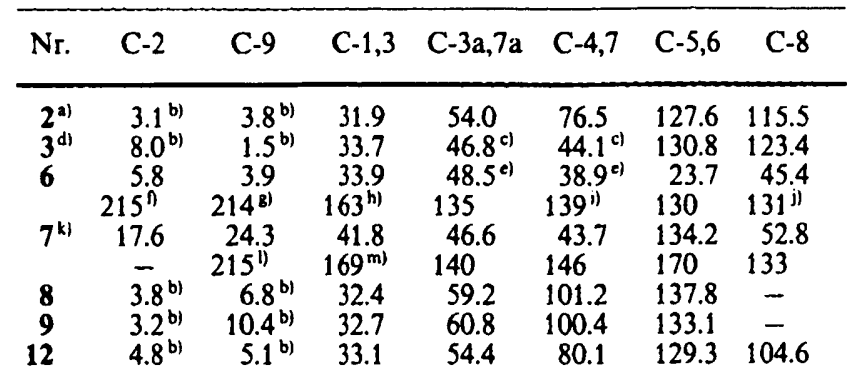

a) 51.4 und $52.3\left(2 \mathrm{CH}_{3}\right)$ - ${ }^{\text {b.c }}$ Zuordnung austauschbar. - d) 49.4 und $51.4\left(2 \mathrm{CH}_{3}\right)$. - ${ }^{e}$ Die Zuordnung dieser Signale stützt sich auf ${ }^{1} J_{C, H}$ aufgrund des Vergleichs mit den entsprechenden Werten von Norbornen: H.O. Kalinowski, S. Berger, S. Braun, ${ }^{13}$ C-NMR-Spektroskopie. Thieme, Stuttgart 1984. Auf der Basis der NorbornenWerte müBte auch die Zuordnung der Banden von $\mathrm{C}-3 \mathrm{a}, 7 \mathrm{a}$ und $C-4,7$ in $5^{7 \mathrm{a})}$ vertauscht werden. - ${ }^{7}$ Feinstruktur $\mathrm{d}, J_{\mathrm{C}-2.9 \cdot \mathrm{H}}=4$. B) Feinstruktur td, $J_{C-9,3-\mathrm{H}}=8, J_{\mathrm{C}-9,2-\mathrm{H}}=4 .{ }^{\text {h) }}$ Feinstruktur d, $J_{\mathrm{C}-1,3-\mathrm{H}}=13 .-$ i) Feinstruktur d, $8 \mathrm{~Hz}$ - - ") Feinstruktur $t$, $8 \mathrm{~Hz}$ - k) $51.4 \mathrm{mit}{ }^{1} J_{\mathrm{C}, \mathrm{H}}=146\left(\mathrm{CH}_{3}\right), 173.3(\mathrm{C}=\mathrm{O})$. - "Feinstruktur $\mathrm{t}, J_{\mathrm{C}-9,3 \mathrm{3}-\mathrm{H}}=8 .{ }^{-{ }^{\mathrm{m})}}$ Feinstruktur $\mathrm{d}, J_{\mathrm{C}-1,3-\mathrm{H}}=14$.

$(1 \alpha, 2 \alpha, 2 a \beta, 2 b \beta, 3 \alpha, 4 \beta, 5 a \beta, 6 \alpha, 6 a \beta)-2 b, 3,4,5 a-T e t r a c h l o r o c t a h y d r o-$ 1-phenylthio-2,4,6-metheno- $1 \mathrm{H}$-cyclobuta[4.5]cyclopenta/b]furan (11) aus 9. - a) Durch Erhitzen mit Thiophenol und AIBN: Analog zur Vorschrift für 10 wurde $11(24 \%)$ als farbloses Ol erhalten, das beim Anreiben mit Petrolether Kristalie mit Schmp. 96-99 $\mathrm{C}$ lieferte.

b) Durch Belichtung mit Thiophenol: Die mit Stickstoff gesättigte Lösung von $2.90 \mathrm{~g}$ (10.2 mmol) 9 und $2.24 \mathrm{~g}$ (20.4 mmol) Thiophenol in $150 \mathrm{ml}$ Benzol wurde $24 \mathrm{~h}$ mit einer Hg-Mitteldrucklampe bei Raumtemp. belichtet. Die Aufarbeitung erfolgte wie bei 10; Ausb. an $1121 \%$. - IR (KBr): $1582\left(\mathrm{C}_{6} \mathrm{H}_{5}\right) \mathrm{cm}^{-1}$. - MS $(70 \mathrm{eV})$ : $m / z(\%)=398,396,394,392\left(4,16,31,22, \mathrm{M}^{+}\right), 221(42), 219(39)$, 185 (74), 183 (100), 149 (76), 147 (67), 115 (52), 109 (80), 77 (39), 65 (40), 39 (47). - 'H-NMR: Tab. 3. - ${ }^{13}$ C-NMR: Tab. 4.

$(1 \alpha, 2 \alpha, 2 a \beta, 2 b \beta, 3 \alpha, 4 \beta, 5 a \beta, 6 \alpha, 6 a \beta)-2 b, 3,4,5,5,5 a-$ Hexachlordecahydro-1-phenylthio-2,4,6-methenocyclobutala/pentalen (13) und (1a $\alpha$, $2 \beta, 2 a \beta, 3 \alpha, 6 \alpha, 6 a \beta, 6 b \alpha)-2,3,4,5,6,7,7-H e p t a c h l o r-1,1 a, 2,2 a, 3,6,6 a, 6 b-$ octahydro-3,6-methanocyclopropa/a]inden (14): $1.60 \mathrm{~g}(4.56 \mathrm{mmol})$ $12^{7 \mathrm{~m}}$, eine Spatelspitze AIBN und $580 \mathrm{mg}$ (5.27 mmol) Thiophenol wurden in $6 \mathrm{ml}$ Benzol $14 \mathrm{~d}$ unter Rückfluß erhitzt, wobei man nach $7 \mathrm{~d}$ weitere $300 \mathrm{mg}(2.73 \mathrm{mmol})$ Thiophenol zugab. Danach extrahierte man das Gemisch mit $2 \mathrm{~N} \mathrm{NaOH}$, dann mit Wasser, trocknete mit $\mathrm{Na}_{2} \mathrm{SO}_{4}$ und verdampfte das Lösungsmittel i.Vak. Der schwarzbraune ölige Rückstand enthielt 13 und 14 im Verhältnis 1:1. Die Hauptmenge des mitentstandenen Diphenyldisulfids trennte man durch Chromatographie $\left(\mathrm{SiO}_{2}, \mathrm{Cyclohexan/Essigester,}\right.$ 12:1) ab. Destillation des übriggebliebenen Gemisches lieferte bei $120-130^{\circ} \mathrm{C}(\mathrm{Bad}) / 0.001$ Torr $600 \mathrm{mg}$ einer Fraktion, die 14 und Diphenyldisulfid im Verhältnis 2:1 und daneben in geringer Menge unbekannte Verunreinigungen enthielt; bei $150-170^{\circ} \mathrm{C}(\mathrm{Bad}) / 0.001$ Torr gingen $480 \mathrm{mg}(23 \%)$ weitgehend reines 13 als farblose Flüssigkeit über, die zu Kristallen mit Schmp. $88-90^{\circ} \mathrm{C}$ erstarrte.

13: IR (KBr): $1578 \mathrm{~cm}^{-1}\left(\mathrm{C}_{6} \mathrm{H}_{5}\right)$ - - MS $(70 \mathrm{eV}): \mathrm{m} / z(\%)=466$, $464,462,460,458\left(1,3,8,10,5, \mathrm{M}^{+}\right), 218(25), 123(43), 113(25)$, 110 (36), 109 (46), 79 (100), 77 (38), 57 (25). - ${ }^{1}$ H-NMR: Tab. 3. ${ }^{13} \mathrm{C}$-NMR: Tab. 4.

$$
\mathrm{C}_{17} \mathrm{H}_{12} \mathrm{Cl}_{6} \mathrm{~S} \text { (461.1) Ber. C } 44.28 \text { H } 2.62
$$$$
\text { Gef. C } 44.55 \text { H } 2.52
$$ 
14: IR (Film): $1608 \mathrm{~cm}^{-1}(\mathrm{C}=\mathrm{C}) .-\mathrm{MS}(70 \mathrm{eV}): \mathrm{m} / \mathrm{z}(\%)=392$, $390,388,386,384,\left(0.4,1.3,2.9,2.9,1.3, \mathrm{M}^{+}\right), 114(20), 113(16), 101$ (17), 79 (100), 77 (38). - 'H-NMR: Tab. 5. - ${ }^{13}$ C-NMR: Tab. 6.

$(1 \alpha, 2 \alpha, 2 a \beta, 2 b \beta, 3 \beta, 4 \beta, 5 a \beta, 6 \alpha, 6 a \beta)-2 b, 3,4,5 a-$ Tetrachlordecahydro5,5-dimethoxy-1-phenylthio-2,4,6-methenocyclobutala a pentalen (15): $1.70 \mathrm{~g}$ (4.97 mmol) 2 und $560 \mathrm{mg}(5.09 \mathrm{mmol})$ Thiophenol wurden in $7 \mathrm{ml}$ Benzol $3 \mathrm{~d}$ unter Rückfluß erhitzt. Danach extrahierte man das Gemisch mit $2 \mathrm{~N} \mathrm{NaOH}$, dann mit Wasser, trocknete mit $\mathrm{Na}_{2} \mathrm{SO}_{4}$ und engtc i.Vak. ein. Umlösen des festen Rückstandes aus Chloroform $/ n$-Hexan führte zu $1.48 \mathrm{~g}(66 \%) 15$ als farblose Kristalle mit Schmp. $138-140^{\circ} \mathrm{C}$. Anstelle des Rückflußkochens erbrachte auch viertägiges Bestrahlen mit 350-nm-Licht (GräntzelReaktor 400) bei Raumtemp. nach Zusatz einer Spatelspitze AIBN das gleiche Ergebnis. Ebenso wandelte sich 2 beim Belichten in reinem Thiophenol ausschließlich in $15 \mathrm{um}$. - IR (KBr): $1585 \mathrm{~cm}^{-1}$ $\left(\mathrm{C}_{6} \mathrm{H}_{6}\right) .-\mathrm{MS}(70 \mathrm{eV}): m / z(\%)=456,454,452,450(4,15,28,21$, $\mathrm{M}^{+}$), 345 (49), 343 (100), 341 (76), 271 (36), 269 (57), 109 (96), 59 (46). - 'H-NMR: Tab. 3. - ${ }^{13} \mathrm{C}-\mathrm{NMR}$ : Tab. 4.

$$
\begin{array}{lll}
\mathrm{C}_{19} \mathrm{H}_{18} \mathrm{Cl}_{4} \mathrm{O}_{2} \mathrm{~S}(452.2) & \text { Ber. C } 50.46 \mathrm{H} 4.01 \\
& \text { Gef. C } 50.33 \text { H } 4.18
\end{array}
$$

[1.2a- $\left.D_{2}\right]$-Derivat 15a: Darstellung aus 2a anhand der Vorschrift für 15. Im ${ }^{1}$ H-NMR-Spektrum fehlen im Vergleich zum Spektrum von 15 die Signale bei $\delta=2.67(2 \mathrm{a}-\mathrm{H})$ und $3.65(1-\mathrm{H})$, und die Multiplizitäten der Banden bei 3.02, 3.18 und 3.53 sind der Erwartung entsprechend reduziert.

$(1 \alpha, 2 \alpha, 2 a \beta, 2 b \beta, 4 \alpha, 5 a \beta, 6 \alpha, 6 a \beta)$-Decahydro-5,5-dimethoxy-1-phenylthio-2,4,6-methenocyclobuta/a]pentalen (16) und $(1 \alpha, 2 \alpha, 3 \alpha, 3 a \beta$. $4 \beta, 5 \beta, 7 \beta, 7 a \beta)-2,3,3 a, 4,5,6,7,7 a$-Octahydro-8,8-dimethoxy-5-phenylthio-4,7-methano-1,2,3-metheno-1H-inden (17): $410 \mathrm{mg}$ (2.01 $\mathrm{mmol})$ $3,230 \mathrm{mg}$ (2.09 mmol) Thiophenol und eine Spatelspitze AIBN wurden bei Raumtemp. in $5 \mathrm{ml}$ Benzol $28 \mathrm{~h}$ mit 350-nm-Licht bestrahlt. Nach Einengen des Gemisches i.Vak. destillierten bei 100 bis $120^{\circ} \mathrm{C}$ (Bad)/0.001 Torr $220 \mathrm{mg}(35 \%)$ einer gelblichen Flüssigkeit aus dem Rückstand, die sich aufgrund des NMR-Spektrums als 1:1-Gemisch aus 16 und 17 erwies. Bei der erneuten, unvollständigen Destillation war 17 im Destillat angereichert, während aus dem Rückstand nach Aufnehmen in Ether und Kühlen auf $-75^{\circ} \mathrm{C} 105 \mathrm{mg}(17 \%) 16$ kristallisierten, Schmp. $60-62^{\circ} \mathrm{C}$. RückfluBkochen von 3 und Thiophenol in Benzol erbrachte nach $12 \mathrm{~h}$ das gleiche Resultat.

16: IR (KBr): $1583 \mathrm{~cm}^{-1}\left(\mathrm{C}_{6} \mathrm{H}_{5}\right)$. - MS $(70 \mathrm{eV}): \mathrm{m} / \mathrm{z}(\%)=314$ $\left(13, \mathbf{M}^{+}\right), 206(17), 205(100), 173(20), 128(12), 115(13), 109(28)$, 101 (14), 97 (12), 91 (19), 77 (16), 65 (15). - 'H-NMR: Tab. 3.

$$
\begin{array}{lll}
\mathrm{C}_{99} \mathrm{H}_{22} \mathrm{O}_{2} \mathrm{~S} \text { (314.5) } & \text { Ber. C } 72.57 \text { H } 7.05 \\
& \text { Gef. C } 72.57 \text { H } 7.04
\end{array}
$$

17: ' $\mathrm{H}-\mathrm{NMR}\left(\mathrm{CDCl}_{3}\right): \delta=3.24,3.29$ (jeweils s; $2 \mathrm{CH}_{3}$ ), 4.03 (dd, $\left.J_{5,6 \alpha}=9.0, J_{5,6 \beta}=5.1 \mathrm{~Hz} ; 5-\mathrm{H}\right), 7.0-7.4\left(\mathrm{~m} ; \mathrm{C}_{6} \mathrm{H}_{5}\right)$, die Lage der weiteren Banden wurde wegen UUberlagerung durch Signale von 16 nicht ermittelt.

$(1 \alpha, 2 \alpha, 3 \alpha, 3 a \beta, 4 \beta, 5 \beta, 7 \beta, 7 a \beta)-2,3,3 a, 4,5,6,7,7 a-$ Octahydro-5-phenylthio-4,7-methano-1,2,3-metheno-1H-inden (18): $1.00 \mathrm{~g} \quad(6.95$ mmol) $5^{72}, 830 \mathrm{mg}$ (7.55 mmol) Thiophenol und eine Spatelspitze AIBN wurden in $7 \mathrm{ml}$ Benzol bei Raumtemp. $24 \mathrm{~h}$ mit $350-\mathrm{nm}$ Licht bestrahlt. Danach extrahierte man das Gemisch mit $2 \mathrm{~N}$ $\mathrm{NaOH}$, dann mit Wasser, trocknete mit $\mathrm{Na}_{2} \mathrm{SO}_{4}$ und engte i. Vak. ein. Aus dem Rückstand destillierten bei $100-105^{\circ} \mathrm{C}(\mathrm{Bad}) / 0.001$ Torr $1.03 \mathrm{~g}(58 \%) 18$ als farblose Flüssigkeit. - IR (Film): 1588 $\mathrm{cm}^{-1}\left(\mathrm{C}_{6} \mathrm{H}_{5}\right) .-\mathrm{MS}(70 \mathrm{eV}): \mathrm{m} / 2(\%)=254\left(31, \mathrm{M}^{+}\right), 174(99)$, 145 (32), 117 (30), 110 (35), 91 (39), 79 (100), 77 (33), 67 (94), 65 (33). $-{ }^{\prime} \mathrm{H}-\mathrm{NMR}\left(\mathrm{CDCl}_{3}\right): \delta=1.23\left(\mathrm{dt}, J_{6,6}=13.0, J_{5,6 \beta}=J_{6 \beta, 7}=\right.$ $4.2 \mathrm{~Hz} ; 6-\mathrm{H}_{\beta}$ ), 1.48 (br. d, $\left.J_{8.8}=10.2 \mathrm{~Hz} ; 8-\mathrm{H}_{a n t i}\right), 1.7-2.3$ (m mit ausgeprägten Maxima bei $1.85,1.98$ und $2.13 ; 1-\mathrm{H}, 2-\mathrm{H}, 3-\mathrm{H}, 3 \mathrm{a}-$ $\left.\mathrm{H}, 4-\mathrm{H}, 7-\mathrm{H}, 7 \mathrm{a}-\mathrm{H}, 8-\mathrm{H}_{s y n}, 9-\mathrm{H}\right), 2.43$ (ddd, $J_{5.6 \alpha}=8.1, J_{6 \alpha .8 s y n}=$ $2.3 \mathrm{~Hz} ; 6-\mathrm{H \alpha}$ ), 3.92 (ddd, $\left.J_{5,85 ; n}=1.7 \mathrm{~Hz} ; 5-\mathrm{H}\right), 7.0-7.5\left(\mathrm{~m} ; \mathrm{C}_{6} \mathrm{H}_{5}\right)$.

$$
\begin{array}{lll}
\mathrm{C}_{17} \mathrm{H}_{18} \mathrm{~S} \text { (254.4) } & \text { Ber. C } 80.26 \text { H } 7.13 \\
& \text { Gef. C } 80.22 \text { H } 7.17
\end{array}
$$

(1 $\alpha, 2 \beta, 3 \alpha, 3 a \beta, 4 \beta, 5 \beta, 7 \beta, 7 a \beta)-1,3,3 a, 4,5,6,7,7 a-$ Octahydro-5-phenylthio-4,7-methano-1,2,3-metheno- $2 \mathrm{H}$-inden-2-carbonsäure-methyl-

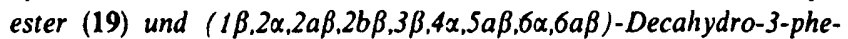
nylthio-2,4,6-methenocylcobuta/ a pentalen-1-carbonsäure-methylester (20). - a) Reaktion von 7 mit Thiophenol in hoher Konzentration: $150 \mathrm{mg}$ ( $0.74 \mathrm{mmol}) 7$ und $20 \mathrm{mg} \mathrm{AIBN} \mathrm{(0.12} \mathrm{mmol)}$ wurden bei $90^{\circ} \mathrm{C} 40 \mathrm{~h}$ in $5 \mathrm{ml}(48.9 \mathrm{mmol})$ Thiophenol gerührt, wobei nach $20 \mathrm{~h}$ weitere $20 \mathrm{mg}$ AIBN zugesetzt wurden. Nach Abkühlen und Zugabe von $50 \mathrm{ml}$ Ether extrahierte man das Gemisch dreimal mit je $35 \mathrm{ml} 2 \mathrm{~N} \mathrm{NaOH}$, dann mit $25 \mathrm{ml}$ Wasser, trocknete mit $\mathrm{Na}_{2} \mathrm{SO}_{4}$ und engte i. Vak. ein. Aus dem Rückstand destillierten bei $50-90^{\circ} \mathrm{C}(\mathrm{Bad}) / 0.001$ Torr ein Vorlauf und bei $150-160^{\circ} \mathrm{C}$ (Bad)/0.001 Torr $130 \mathrm{mg}(56 \%)$ eines farblosen Ols, dessen NMRSpektren ein 1.0:0.4-Gemisch aus 19 und 20 anzeigten. Durch Chromatographie $\left(\mathrm{SiO}_{2}\right.$, Cyclohexan/Essigester, 5:1, Chromatotron) wurden $60 \mathrm{mg}(26 \%) 19$ und $25 \mathrm{mg}(11 \%)$ noch etwas. verunreinigtes 20 erhalten, jeweils als Öl. Zwei Versuche, bei denen das Thiophenol ein wenig mit Benzol verdünnt war, erbrachten kleinere Verhältnisse 19:20 (siehe Abschnitt B).

b) Reaktion von 7 mit Thiophenol in niedriger Konzentration: $150 \mathrm{mg}(0.74 \mathrm{mmol}), 20 \mathrm{mg}(0.12 \mathrm{mmol})$ AIBN und $90 \mathrm{mg}(0.82$ mmol) Thiophenol wurden in $500 \mathrm{ml}$ Benzol 65 h unter Rückfluß erhitzt, wobei nach $32 \mathrm{~h}$ weitere $20 \mathrm{mg}$ AIBN zugesetzt wurden. Die Aufarbeitung erfolgte wie unter a). Durch Chromatographie wurden $25 \mathrm{mg}(11 \%)$ noch etwas verunreinigtes 20 erhalten. Das ${ }^{1}$ H-NMR-Spektrum des Rohprodukts zeigt keine Signale von 19 und erlaubt die Aussage: 19:20 $\leqq 1: 18$.

19: IR $\left(\mathrm{CCl}_{4}\right)$ : $1718(\mathrm{C}=\mathrm{O}), 1585 \mathrm{~cm}^{-1}\left(\mathrm{C}_{6} \mathrm{H}_{5}\right)$. - 'H.NMR $\left(\mathrm{CDCl}_{3}\right): \delta=1.27$ (dtd, $J_{6,6}=13.2, J_{5,6 \beta}=J_{6 \beta, 7}=4.5, J_{6 \beta, 7 \mathrm{a}} \approx$ $\left.1 \mathrm{~Hz} ; 6-\mathrm{H}_{\beta}\right), 1.49\left(\approx\right.$ dquint, $J_{8,8}=10.0, J_{6 \alpha, 8 s y n}=2.5, J_{4,85 y n}=$ $\left.J_{5,8 s y n}=J_{7,8 s y n}=1.7 \mathrm{~Hz} ; 8-\mathrm{H}_{s y n}\right), 1.87\left(\mathrm{dt}, J_{4,8 a n t i}=J_{7,8 a n t i}=\right.$ $\left.1.5 \mathrm{~Hz} ; 8-\mathrm{H}_{a n t i}\right), 2.19(\mathrm{~m} ; 4-\mathrm{H}, 7-\mathrm{H}), 2.25\left(\mathrm{ddd}, J_{3.6 x}=8.2 \mathrm{~Hz}\right.$; 6- $\left.\mathrm{H}_{\alpha}\right), 2.30\left(\mathrm{ddq}, J_{3 \mathrm{a}, 7_{\mathrm{a}}}=9.5, J_{7,7 \mathrm{a}}=4.5, J_{1,7 \mathrm{a}} \approx J_{7 \mathrm{a}, 9} \approx 1 \mathrm{~Hz}\right.$; $7 \mathrm{a}-\mathrm{H}$ ), 2.37 (ddt, $J_{3 \mathrm{a}, 4}=4.7, J_{3,3 \mathrm{a}} \approx J_{3 \mathrm{a}, 9} \approx 1 \mathrm{~Hz} ; 3 \mathrm{a}-\mathrm{H}$ ), 2.68 und 2.73 (jeweils dm, $J_{1,3} \approx 5 \mathrm{~Hz} ; 1-\mathrm{H}, 3-\mathrm{H}$ ), 2.80 (tt, $J_{1,9}=J_{3,9}=2.0$ $\mathrm{Hz}$; 9-H), 3.67 (s; $\left.\mathrm{CH}_{3}\right), 3.69$ (ddd; 5-H), $\mathrm{C}_{6} \mathrm{H}_{5}: 7.15(\mathrm{p}-\mathrm{H}), 7.26(o-$ $\mathrm{H}, m-\mathrm{H}) .-{ }^{13} \mathrm{C}-\mathrm{NMR}\left(\mathrm{CDCl}_{3}\right): \delta=19.1(\mathrm{~s} ; \mathrm{C}-2), 20.6(\mathrm{~d}, 211 \mathrm{~Hz}$; C-9), 32.9 (t, $132 \mathrm{~Hz}), 41.8$ (t) (C-6, C-8), 38.5, 39.8, 40.4, 42.5, 44.4, 46.5, 47.3 (jeweils d; C-1, C-3, C-3a, C-4, C-5, C-7, C-7a); 51.6 (q; $\left.\mathrm{CH}_{3}\right) ; 172.0$ (s; C=O); $\mathrm{C}_{6} \mathrm{H}_{5}: 125.4(p-\mathrm{C}) ; 128.4,128.7$ (o-C, $\left.m-\mathrm{C}\right)$, 137.8 (ipso-C).

20: IR (Film): $1730(\mathrm{C}=\mathrm{O}), 1583 \mathrm{~cm}^{-1}\left(\mathrm{C}_{6} \mathrm{H}_{5}\right) .-{ }^{1} \mathrm{H}-\mathrm{NMR}$ : Tab. 3. - ${ }^{3}$ C-NMR: Tab. 4.

Gemisch aus 19 und $20(1.0: 1.7):$ MS $(70 \mathrm{eV}): m / z(\%)=312$ $\left(13, \mathrm{M}^{+}\right), 186(89), 185(59), 143(40), 128(31), 105(68), 79(74), 77$ (81), $66(100), 65(39), 51(45)$.

$$
\begin{array}{lll}
\mathrm{C}_{19} \mathrm{H}_{20} \mathrm{O}_{2} \mathrm{~S} \text { (312.4) } & \text { Ber. C } 73.04 \text { H } 6.45 \\
& \text { Gef. C } 73.24 \text { H } 6.44
\end{array}
$$

\section{Addition von Bromtrichlormethan an 12}

$(1 \alpha, 2 \alpha, 2 a \beta, 2 b \beta, 3 \beta, 4 \beta, 5 a \beta, 6 \alpha, 6 a \beta)-3-B r o m-2 b, 3,4,5,5,5 a$-hexachlordecahydro-1-trichlormethyl-2,4,6-methenocyclobutala]pentalen (21): $1.00 \mathrm{~g}$ (2.84 mmol) 12 wurden in $40 \mathrm{ml}$ trockenem Hexan und $60 \mathrm{ml}(610 \mathrm{mmol})$ Bromtrichlormethan bei Raumtemp. $3 \mathrm{~h}$ mit einer Quecksilberdampflampe (Hanau TQ 150) durch Pyrexglas bestrahlt. Anschließend wurde i.Vak. vollständig eingeengt, der Rückstand mit Dichlormethan/Hexan zur Kristallisation gebracht und 
mit diesem Lösungsmittelgemisch umgelöst: $830 \mathrm{mg}$ (53\%) 21 als farblose Kristalle mit Schmp. $165-167^{\circ} \mathrm{C}$. - MS (70 eV): $\mathrm{m} / \mathrm{z}$ $(\%)=554,552,550,548,546,544,\left(0.5,0.5,0.8,0.8,0.4,0.1, \mathrm{M}^{+}\right)$, 299 (82), 297 (100), 295 (55), 256 (40), 254 (43), 229 (53), 220 (46),

Tab. 3. 'H-NMR-Chemische Verschiebungen ( $\delta$-Werte) und Kopplungskonstanten $[\mathrm{Hz}]$ von Decahydro-2,4,6-methenocyclobuta[a]pentalen-Derivaten in $\mathrm{CDCl}_{3}$. Die generell angegebenen Multiplizitäten sind nur bei Abwesenheit in Effekten höherer Ordnung zu beobachten. Die Absorptionen der Phenylthioreste liegen bei $7.18-7.23(p-H)$ und $7.22-7.33(m-\mathrm{H}, o-\mathrm{H}$; in einigen Fällen ist das $o$-H-Signal als solches bei tieferem Feld erkennbar). Die Kopplungskonstanten variieren nur wenig: $J_{1.2}=J_{1,6 \mathrm{a}}=J_{2.2 \mathrm{a}}=2.7-3.0$ (Ausnahme 20 bezüglich $J_{1,2}$ und $J_{1,6 a}$, siche Abschnitt B), $J_{2 a, 6}=$ $J_{2,7}=1.5-2.2$ (Ausnahme 21: 2.9), $J_{2.62}=5.5-6.0$ (Ausnahme 21: 5.0), $J_{6.7}=6.5-7.2, J_{2.7}=1.0-1.3$ (Ausnahme 21: 1.9), $J_{6,6 \mathrm{a}}=$ $1.7-2.2$

\begin{tabular}{|c|c|c|c|c|c|c|c|}
\hline Nr. & $\underset{t}{t-H}$ & $\begin{array}{l}2 \mathrm{a}-\mathrm{H} \\
\approx \text { quint }\end{array}$ & $\begin{array}{c}\text { 6a-H } \\
\text { dtd }\end{array}$ & $\underset{\text { br.dtd }}{2-H}$ & $\begin{array}{l}6-\mathrm{H} \\
\mathrm{dm}\end{array}$ & $\begin{array}{l}\text { 7-H } \\
\mathrm{dm}\end{array}$ & $3-\mathrm{H}$ \\
\hline $\begin{array}{l}11 \\
13 \\
15^{c)} \\
16^{()}\end{array}$ & $\begin{array}{l}3.89 \\
3.72 \\
3.65 \\
3.58\end{array}$ & $\begin{array}{l}2.72 \\
2.77 \\
2.67\end{array}$ & $\begin{array}{l}3.05 \\
3.17 \\
3.18 \\
\text { e) }\end{array}$ & $\begin{array}{l}3.48 \\
3.66 \\
3.53\end{array}$ & $\begin{array}{l}3.39 \\
3.36^{b 1} \\
3.02\end{array}$ & $\begin{array}{l}3.37 \\
3.13^{b)} \\
3.02 \\
e^{b}\end{array}$ & $\begin{array}{l}4.12^{\mathrm{a}} \\
5.01^{\mathrm{a}} \\
4.66^{\mathrm{d}} \\
\text { e) }\end{array}$ \\
\hline $\begin{array}{l}20^{n} \\
21\end{array}$ & $\begin{array}{l}3.15^{81} \\
4.33\end{array}$ & $\begin{array}{l}2.60^{h)} \\
3.14\end{array}$ & $\begin{array}{l}2.68^{\mathrm{i}} \\
3.33^{\mathrm{kl}}\end{array}$ & $\begin{array}{l}2.72^{i)} \\
3.81^{k 1}\end{array}$ & $\begin{array}{l}2.44 \\
3.62^{\mathrm{k})}\end{array}$ & $\begin{array}{l}2.38 \\
3.36^{k)}\end{array}$ & $3.26^{\mathrm{j}}$ \\
\hline
\end{tabular}

a) $\mathrm{d}, J_{3,7}=1.0$. $-{ }^{\text {b) }}$ Die Zuordnung wurde durch Entkopplung von 3-H gesichert. - c) 3.60 (s), 3.63 (s) $\left(2 \mathrm{CH}_{3}\right)$ - d) s. - el 1.00 (dd, $\left.J_{3,3}=10.8, J_{2 \mathrm{~b}, 3 \alpha}=1.7 ; 3-\mathrm{H}_{\alpha}\right), 1.58\left(\right.$ ddd, $J_{2 \mathrm{~b}, 3 \beta}=5.8, J_{3 \beta, 4}=1.9$ $\left.3-\mathrm{H}_{\beta}\right), 1.9-2.3(\mathrm{~m} ; 2 \mathrm{H}), 2.3-2.8(\mathrm{~m} ; 6 \mathrm{H}), 3.23(\mathrm{~s})$ und $3.28(\mathrm{~s})$ $\left(2 \mathrm{CH}_{3}\right) .-1.66$ (br.d, $\left.J_{5.5}=10.5 ; 5-\mathrm{H}_{\beta}\right), 2.06\left(\mathrm{tt}, J_{2 \mathrm{~b}, \mathrm{~s}_{\mathrm{a}}}=J_{5 \mathrm{a} .6}=\right.$
$\left.5.9, J_{5 \alpha .5 \mathrm{a}}=J_{5 \mathrm{p} . \mathrm{s}_{\mathrm{a}}}=1.5 ; 5 \mathrm{a}-\mathrm{H}\right), 2.17$ (br.dd, $\left.J_{3.5 \mathrm{x}}=2.5 ; 5-\mathrm{H}_{x}\right), 2.33$ (m; 2b-H), 2.53 (br.s; 4-H), $3.68\left(\mathrm{~s} ; \mathrm{CH}_{3}\right) .-{ }_{\mathrm{g}} \mathrm{J}=1.5 \mathrm{~Hz}$, Kopplungspartner sind möglicherweise $2 \mathrm{a}-\mathrm{H}$ und $2 \mathrm{~b}-\mathrm{H} .{ }^{\left.-{ }^{h}\right)} \mathrm{dm}, J_{2 \mathrm{a}, 2 \mathrm{~b}} \approx$ 5.5. - i) ddd. - il Breit. - k) Durch Entkoppeln sind die Nachbarschaftsbeziehungen gesichert, jedoch ist die Vertauschung der Paare 2-H, 7-H und 6- $\mathrm{H}, 6 \mathrm{a}-\mathrm{H}$ möglich.

Tab. 4. ${ }^{13} \mathrm{C}$-NMR-Chemische Verschiebungen ( $\delta$-Werte) von Decahydro-2,4,6-methenocyclobuta [a]pentalen-Derivaten in $\mathrm{CDCl}_{3}$ und bei 21 auch ${ }^{13} \mathrm{C},{ }^{1} \mathrm{H}-\mathrm{K}$ opplungskonstanten $\left(\mathrm{Hz},{ }^{1} J_{\mathrm{C}, \mathrm{H}}\right.$ in der zweiten Zeile). Bei der Zuordnung halfen off-resonance-entkoppelte Spektren. Die Absorptionen der Phenylthioreste liegen bei 125.9-127.1 $(p-C), 128.7-129.3(m-C), 129.5-130.4(o-C)$ und 134.4-136.7 (ipso-C)

\begin{tabular}{|c|c|c|c|c|c|}
\hline & \multirow{2}{*}{$\begin{array}{l}\text { Nr. } \\
11 \\
13 \\
15^{c)} \\
20^{d)} \\
21^{(c)}\end{array}$} & \multicolumn{3}{|c|}{$C-1, C-2, C-2 a, C-6, C-6 a, C-7^{21}$} & \\
\hline & & $\begin{array}{l}44.0,48.2 \\
45.3,48.8, \\
46.0,47.8, \\
43.5,43.6, \\
44.8,45.4 \\
166,160\end{array}$ & $\begin{array}{l}53.2,53.5, \\
49.1,49.5, \\
47.9,49.3, \\
44.1,46.0 \\
51.1,59.8, \\
164156\end{array}$ & $\begin{array}{ll}53.6, & 59.5 \\
56.0, & 57.1 \\
50.8, & 55.6 \\
48.0, & 50.2 \\
63.7, & 64.2 \\
160 & 160\end{array}$ & \\
\hline Nr. & C-5a & $C-2 b$ & $\mathrm{C}-3$ & C-4 & C-5 \\
\hline $\begin{array}{l}11 \\
13 \\
15^{\mathrm{c}} \\
\left.20^{\mathrm{d}}\right) \\
21^{\mathrm{c}}\end{array}$ & $\begin{array}{l}109.2 \\
88.4 \\
84.0^{b} \\
51.3^{\text {b) }} \\
90.1^{\text {b) }} \\
-\end{array}$ & $\begin{array}{l}74.1^{\text {b) }} \\
74.0^{\text {b) }} \\
76.6^{\text {b) }} \\
53.4^{\text {b) }} \\
82.9^{\text {b) }} \\
-\end{array}$ & $\begin{array}{l}70.8 \\
69.7 \\
69.2 \\
51.7^{b)} \\
82.6^{b)} \\
-\end{array}$ & $\begin{array}{l}96.4^{b} \\
75.3^{b)} \\
69.9^{b)} \\
39.8^{b)} \\
91.5^{b)} \\
-\end{array}$ & $\begin{array}{r}\overline{97.1} \\
104.4 \\
36.6 \\
94.9 \\
-\end{array}$ \\
\hline
\end{tabular}

a) Die spezifische Zuordnung der Signale zu diesen C-Atomen ist nicht möglich. - b) Zuordnung austauschbar. - c) $51.3,51.8$, $\left(2 \mathrm{CH}_{3}\right)$, Zuordnung mit der eines Teils der anderen Banden austauschbar. - di $51.4\left(\mathrm{CH}_{3}\right)$, Zuordnung mit der eines Teils der anderen Banden alustauschbar: $173.8(\mathrm{C}=\mathrm{O})$. ${ }^{\circ} 102.2\left(\mathrm{CCl}_{3}\right)$.
$119(60), 117$ (58), 113 (88), 77 (48). - 'H-NMR: Tab. 3. - ${ }^{13} \mathrm{C}$ VMR: Tab. 4.

$$
\begin{array}{lllll}
\mathrm{C}_{12} \mathrm{H}_{6} \mathrm{BrCl}_{9}(549.2) & \text { Ber. C } 26.24 & \mathrm{H} 1.10 \\
\ldots & \text { Gef. C } 26.40 & \text { H } 1.17
\end{array}
$$

\section{Additionen von Säuren an 3, 5 und 12}

Essigsäure-( ( $1 a \alpha, 2 \beta, 2 a \beta, 3 \alpha, 6 \alpha, 6 a \beta, 6 b \alpha)-3,4,5,6,7,7$-hexachlor1, Ia.2,2a,3,6.6a,6b-octahydro-3,6-methanocyclopropa[a /inden-2-yl-

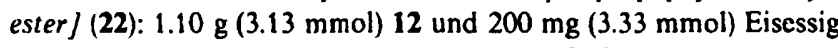
wurden in $5 \mathrm{ml}$ Chloroform $7 \mathrm{~d}$ unter Rückfluß erhitzt. Man verdünnte dann die Lösung mit Ether, neutralisierte durch Extraktion mit $2 \mathrm{~N} \mathrm{NaOH}$, trocknete die Etherphase mit $\mathrm{Na}_{2} \mathrm{SO}_{4}$ und engte i. Vak. ein. Aus dem Rückstand destillierten bei $120-125^{\circ} \mathrm{C}$ (Bad)/ 0.001 Torr $930 \mathrm{mg}$ eines 3.5:1.0-Gemisches aus 22 (ca. 50\%) und einer unbekannten Komponente als farblose Flüssigkeit. Die Trennung gelang nicht. - IR (Film): $1745(\mathrm{C}=\mathrm{O}), 1608 \mathrm{~cm}^{-1}(\mathrm{C}=\mathrm{C})$. - 'H-NMR: Tab. 5. - ${ }^{13}$ C-NMR: Tab. 6.

4-Nitrobenzoesäure-( ( $1 a \alpha, 2 \beta, 2 a \beta, 3 \beta, 6 \beta, 6 a \beta, 6 b \alpha)-1,1 a, 2,2 a, 3,6$, $6 a, 6 b$-octahydro-7,7-dimethoxy-3,6-methanocyclopropa[a /inden-2ylester] (23): Die Suspension von $650 \mathrm{mg}$ (3.89 mmol) 4-Nitrobenzoesäure in der Lösung von $800 \mathrm{mg}$ (3.92 mmol) 3 in $15 \mathrm{ml} \mathrm{Chlo-}$ roform wurde $10 \mathrm{~d}$ bei $20^{\circ} \mathrm{C}$ gerührt. Dann engte man i.Vak. ein und nahm den öligen Rückstand in heißem Ethanol auf. Nach dem Abkühlen schieden sich $1.05 \mathrm{~g}(73 \%) 23$ als farblose Kristalle mit Schmp. $139-141^{\circ} \mathrm{C}$ ab. - IR $(\mathrm{KBr}): 1710(\mathrm{C}=\mathrm{O}), 1607(\mathrm{C}=\mathrm{C})$, $1527 \mathrm{~cm}^{-1}\left(\mathrm{NO}_{2}\right) . \quad$ MS (70 eV): $\mathrm{m} / z(\%)=371(39), 204(47), 189$ (46), 151 (83), 150 (100), 129 (77), 104 (67), 91 (48), 75 (41), 59 (55). ${ }^{1}$ H-NMR: Tab. 5. - ${ }^{13}$ C-NMR: Tab. 6.

$$
\begin{array}{lllll}
\mathrm{C}_{20} \mathrm{H}_{21} \mathrm{NO}_{6} \text { (371.4) } & \text { Ber. C } 64.68 \text { H } 5.70 \text { N } 3.77 \\
& \text { Gef. C } 64.18 \text { H } 5.75 \text { N } 3.36
\end{array}
$$

4-Nitrobenzoesäure- $/(1 a \alpha, 2 \beta, 2 a \beta, 3 \beta, 6 \beta, 6 a \beta, 6 b \alpha)-1,1 a, 2,2 a, 3,6$, 6a.6b-octahydro-3,6-methanocyclopropa [a /inden-2-ylester ] (24): Die Suspension von $1.67 \mathrm{~g}$ (10.0 mmol) 4-Nitrobenzoesäure in der Lösung von $1.30 \mathrm{~g}$ ( $9.01 \mathrm{mmol}) 5$ in $20 \mathrm{ml}$ Chloroform wurde $14 \mathrm{~d}$ bei $20^{\circ} \mathrm{C}$ gerührt. Durch Aufarbeitung wie bei 24 fielen $2.12 \mathrm{~g}(76 \%)$ 24 als farblose Kristalle mit Schmp. $90-92^{\circ} \mathrm{C}$ (aus Methanol) an. - IR (KBr): $1708(\mathrm{C}=\mathrm{O}), 1604(\mathrm{C}=\mathrm{C}), 1525 \mathrm{~cm}^{-1}\left(\mathrm{NO}_{2}\right)$. MS $(70 \mathrm{eV}): m / z(\%)=311\left(1, \mathrm{M}^{+}\right), 150(23), 79(32), 78(96), 66$ (100). - 'H-NMR: Tab. 5. $-{ }^{13}$ C-NMR: Tab. 6.

$$
\begin{array}{lllll}
\mathrm{C}_{18} \mathrm{H}_{17} \mathrm{NO}_{4} \text { (311.3) } & \text { Ber. C } 69.44 \text { H } 5.50 \text { N } 4.50 \\
& \text { Gef. C } 69.06 \text { H } 5.85 \text { N } 4.46
\end{array}
$$

Tab. 5. 'H-NMR-Chemische Verschiebungen ( $\delta$-Werte) und Kopplungskonstanten $[\mathrm{Hz}]$ von $1,1 \mathrm{a}, 2,2 \mathrm{a}, 3,6,6 \mathrm{a}, 6 \mathrm{~b}-$ Octahydro-3,6-methanocyclopropa $[a]$ indenen in $\mathrm{CDCl}_{3}$. Die allgemein angegebenen Multiplizitäten sind bei Gleichheit von Kopplungskonstanten vereinfacht. $J_{1 \alpha, 1 \beta}=4.5-5.5, J_{1 \alpha,\}_{\mathrm{a}}}=J_{1 \mathrm{\alpha}, 6 \mathrm{~b}}=8.1-8.4, J_{1 \alpha, 2} \approx$ $J_{1 \alpha, 2 \mathrm{a}} \approx 0.8 ; J_{1 \beta, 1 \mathrm{a}}=J_{1 \beta, 6 \mathrm{~b}}=4.0-4.6, J_{1 \mathrm{a}, 2}=5.4-5.6, J_{1 \mathrm{a}, 6 \mathrm{~b}}=$ $4.8-6.0, J_{2,2 \mathrm{a}}=2.4-3.0, J_{2 \mathrm{a}, 6 \mathrm{a}}=7.3-7.7$

\begin{tabular}{lccccccc}
\hline Nr. & $\begin{array}{c}1 \alpha-\mathrm{H} \\
\text { tdt }\end{array}$ & $\begin{array}{c}1 \beta-\mathrm{H} \\
\mathrm{dt}\end{array}$ & $\begin{array}{c}1 \mathrm{a}-\mathrm{H} \\
\mathrm{dtd}\end{array}$ & $\begin{array}{c}2-\mathrm{H} \\
\mathrm{ddd}\end{array}$ & $\begin{array}{c}2 \mathrm{a}-\mathrm{H} \\
\mathrm{ddd}\end{array}$ & $\begin{array}{c}6 \mathrm{a}-\mathrm{H} \\
\mathrm{d}\end{array}$ & $\begin{array}{c}6 \mathrm{~b}-\mathrm{H} \\
\mathrm{ddd}\end{array}$ \\
\hline $\mathbf{1 4}$ & 0.88 & 0.57 & 1.93 & 4.58 & 3.23 & 3.43 & 1.61 \\
$\mathbf{2 2}^{\text {a) }}$ & 0.68 & 0.43 & 1.92 & 5.22 & 2.98 & 3.32 & 1.47 \\
$\mathbf{2 3}^{\text {b.c) }}$ & 0.56 & 0.46 & 1.63 & 5.13 & $2.52^{\mathrm{d})}$ & $2.88^{\mathrm{e})}$ & 1.11 \\
$\mathbf{2 4}^{\text {(.) }}$ & 0.51 & 0.39 & 1.61 & 5.04 & $2.40^{\mathrm{s}}$ & $2.72^{\mathrm{h}}$ & 1.12 \\
\hline
\end{tabular}

a) $1.98\left(\mathrm{~s} ; \mathrm{CH}_{3}\right)$ - b) $2.93\left(\mathrm{ddt}, J_{2.3}=4.5, J_{3,4}=3.1, J_{3.5}=J_{3.6}=\right.$ $1.3 ; 3-\mathrm{H}), 3.10$ (m, überlagert; $6-\mathrm{H}$ ); 3.06 und 3.09 (jeweils s, $2 \mathrm{CH}_{3}$ ), 6.23 und 6.25 (jeweils ddd, $J_{4,5}=6.0 ; 4-\mathrm{H}_{2} 5-\mathrm{H}$ ). $-{ }^{\mathrm{c}} 8.12$ und 8.21 (AA'BB'-Spektrum; 4 aromat. H). - d dddd. - e) dd, $J_{0,6 \mathrm{a}}=$ 4.3. -1.22 und 1.42 (jeweils br.d, $J_{7,7}=8.1 ; 7 \cdot \mathrm{H}_{2}$ ), 2.85 und 3.12 (jeweils br.s; $3-\mathrm{H}, 6-\mathrm{H}), 6.17$ und 6.22 (jeweils dd $J_{4,5}=5.2, J_{3.4}=$ $\left.J_{5.6}=2.8 ; 4-\mathrm{H}, 5-\mathrm{H}\right) .-{ }^{8} \mathrm{dddd}, J_{2 \mathrm{~s}, 3}=4.0 .{ }^{h 1} \mathrm{dd}, J_{6,60}=4.0$. 
Tab. 6. ${ }^{13} \mathrm{C}$-NMR-Chemische Verschiebungen ( $\delta$-Werte) von 1,1a,2,2a,3,6,6a,6b-Octahydro-3,6-methanocyclopropa[a]indenen in $\mathrm{CDCl}{ }_{3}$

\begin{tabular}{lrllllll}
\hline Nr. & C-1 & C-1a, C-6b & C-2 & C-2a, C-6a & C-3, C-6 & C-4, C-5 & C-7 \\
\hline $\mathbf{1 4}^{\text {b) }}$ & 11.3 & $22.5,28.8$ & $58.1^{\text {a) }}$ & $60.3,64.0^{\text {a) }}$ & $80.7,81.0$ & $129.6,131.8$ & 103.4 \\
$\mathbf{2 3}^{\text {(d) }}$ & 7.9 & $25.9,20.3^{\text {a) }}$ & 75.3 & $57.5,59.5$ & $80.4,81.1$ & $129.3,131.5$ & 103.5 \\
$\mathbf{2 4}^{\text {d) }}$ & 8.0 & $22.2,25.6$ & 82.1 & $49.6,50.1^{\text {a) }}$ & $48.1,49.6^{\text {a) }}$ & $131.5,133.9$ & 121.2 \\
\hline
\end{tabular}

a) Zuordnung austauschbar. - b) $20.9^{\text {a) }}\left(\mathrm{CH}_{3}\right), 169.8(\mathrm{C}=\mathrm{O})$. - c) 51.1, 51.9 a) $\left(2 \mathrm{CH}_{3}\right)$. - d) $164.8(\mathrm{C}=\mathrm{O}) ; 4-\mathrm{NO}_{2}-\mathrm{C}_{6} \mathrm{H}_{4}: 123.4(\mathrm{C}-3), 130.5$ (C-2), 136.3 (C-1), $150.5(\mathrm{C}-4)$.

\section{CAS-Registry-Nummern}

1: $659-85-8$ / 1 a: $87986-01-4$ / 2: 115408-13-4 / 2a: 115408-31-6/ 3: $115408-14-5$ / 4: 115408-15-6 / 5: 65282-11-3 / 6: 115461-01-3 / 7: 115408-17-8 / 8: 115509-04-1 / 9: 115408-18-9 / 10: 115408-19-0/ 11: $115408-20-3$ / 12: 65364-45-6 / 13: $115419-78-8$ / 14: 115408 21-4 / 15: 115408-22-5 / 15a: 115408-32-7 / 16: 115408-23-6 / 17: 115408-24-7 / 18: 115408-25-8 / 19: 115408-26-9 / 20: 115408-27-0 / 21: $115419-79-9$ / 22: 115408-28-1 / 23: 115408-29-2 / 24: 11540830-5 / 1,2,3,4-Tetrachlor-5,5-dimethoxycyclopentadien: 2207-27-4 / p-Nitrobenzolsulfonsäurehydrazid: 2937-05-5 / Chlorameisensäuremethylester: 79-22-1 / Tetrachlorfuran: 2763-65-7

"Aus der Diplomarbeit S. Brengel und der Dissertation S. Freund, Univ. Würzburg, 1980 und 1983.

2) Aus der Dissertation H. Henneberger, Univ. Würzburg, 1987.

3) B. Giese, Radicals in Organic Synthesis. Formation of Carbon-Carbon Bonds, Pergamon Press, Oxford 1986.

4) A. L. J. Beckwith, K. U. Ingold in Rearrangements in Ground and Excited States (P. de Mayo, Ed.), Bd. 1, S. 161, Academic Press, New York 1980.

5) sa) G. Szeimies, A. Schloßer, F. Philipp, P. Dietz, W. Mickler, Chem. Ber. 111 (1978) 1922. - sb) P. Dietz, G. Szeimies, Chem.

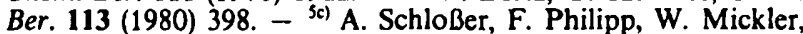
G. Szeimies, H.-D. Martin, Chem. Ber. 113 (1980) 1053.

6) 6a) R. Herbert, M. Christl, Chem. Ber. 112 (1979) 2012. - ${ }^{6 b} \mathrm{H}$ Leininger, F. Lanzendörfer, M. Christl, Chem. Ber. 116 (1983) 669. - ${ }^{6 c)}$ M. Christl, H. Leininger, P. Kemmer, Chem. Ber. 117 (1984) 2963. - ${ }^{6 d)}$ C. Herzog, R. Lang, D. Brückner, P. Kemmer, M. Christl, Chem. Ber. 119 (1986) 3027.

7) 7.) M. Christl, H.-J. Lüddeke, A. Nagyrevi-Neppel, G. Freitag Chem. Ber. 110 (1977) 3745. - b) M. Christl, Angew. Chem. 93 (1981) 515; Angew. Chem., Int. Ed. Engl. 20 (1981) 529. - 7c) M. Christl. S. Freund, Chem. Ber. 118 (1985) 979.

8) G. J. Kent, S. A. Godleski, E. Osawa, P. v. R. Schlcyer, J. Org. Chem. 42 (1977) 3852.

${ }^{9}$ W. R. Moore, S. S. Hall, C. Largman. Tetrahedron Lett. 1969, 4.353 .
10) L. A. Paquette, G. Zon, J. Am. Chem. Soc. 96 (1974) 224.

11) H. Krzikalla, H. Linge, Chem. Ber. 96 (1963) 1751.

12) H. Feichtinger, H.-W. Linden, Chem. Ber. 102 (1969) 3573; T. Jaworski, Rocz. Chem. 43 (1969) 53 [C. A. 70 (1969) 115033a].

13) J. Sauer, Angew. Chem. 79 (1967) 76; Angew. Chem.. Int. Ed. Engl. 6 (1967) 16

14) M. Christl, R. Lang, R. Herbert, G. Freitag, Angew. Chem. 92 (1980) 465; Angew. Chem., Int. Ed. Engl. 19 (1980) 457.

15) P. Bischof, J. A. Hashmall, E. Heilbronner, V. Hornung, Helv. Chim. Acta 52 (1969) 1745.

16) R. Gleiter, Top. Curr. Chem. 86 (1979) 197.

17) Abgeschätzt aus Gruppeninkrementen und Spannungsenergien nach S. W. Benson, Thermochemical Kinetics, 2nd Ed., Wiley, New York 1976; K. B. Wiberg, Angew. Chem. 98 (1986) 312; Angew. Chem., Int. Ed. Engl. 25 (1986) 312.

18) K. B. Wiberg, B. R. Lowry, B. J. Nist, J. Am. Chem. Soc. 84 (1962) 1594.

19) R. Stangl, Dissertation, Univ. Würzburg, 1984.

20) $\mathrm{S}$. Hoz in The chemistry of the cyclopropyl group (Z. Rappoport, Ed.), Part 2, S. 1121, Wiley, Chichester 1987.

21) H. Leininger, P. Kemmer, K. Beck, M. Christl, Chem. Ber. 115 (1982) 3213.

22) P. G. Gassman, J. L. Marshall, Org. Synth., Coll. Vol. V (1973) 424.

23) T. J. Katz, E. J. Wang, N. Acton, J. Am. Chem. Soc. 93 (1971) 3782; T. J. Katz, R. J. Roth, N. Acton, E. J. Carnahan, Org. Synth. 53 (1973) 157. Zur Darstellung von Benzvalen verwendeten wir bromidfreies Methyllithium von CHEMETALL.

24) R. J. Roth, T. J. Katz, J. Am. Chem. Soc. 94 (1972) 4770.

25) Zur Methode vgl. S. Hünig, H. R. Müller, W. Thier, Angew. Chem. 77 (1965) 368; Angew. Chem., Int. Ed. Engl. 4 (1965) 271. W. Thier, Dissertation, Univ. Würzburg, 1964.

26) G. Maaß, Angew. Chem. 77 (1965) 811; Angew. Chem., Int. Ed. Engl. 4 (1965) 787.

27) BASF AG (H. Krzikalla, H. Linge, Erf.) D.B.P. 932612 (05.09.1955), 947310 (16.08.1956) [Chem. Abstr. 52 (1958) $17287 \mathrm{f}, 53$ (1959) 406f]. [R. Stroh in Methoden der organischen Chemie (Houben-Weyl-Müller), 4. Aun., Bd. V/4, S. 718, Thieme, Stuttgart 1960]. 